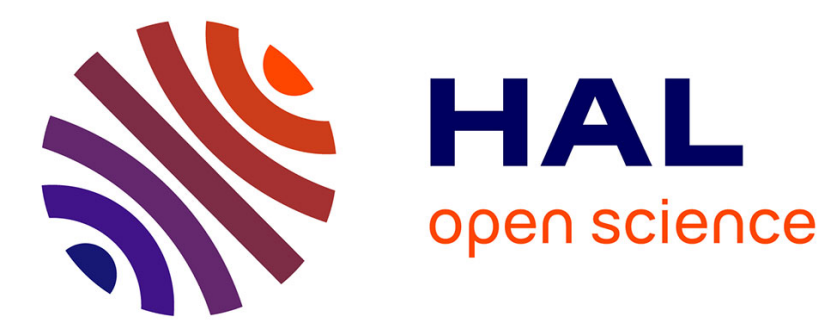

\title{
Study of a numerical scheme for miscible two-phase flow in porous media
}

Robert Eymard, Veronika Schleper

\section{To cite this version:}

Robert Eymard, Veronika Schleper. Study of a numerical scheme for miscible two-phase flow in porous media. 2013. hal-00741425v2

\section{HAL Id: hal-00741425 \\ https://hal.science/hal-00741425v2}

Preprint submitted on 11 Jul 2013 (v2), last revised 1 Mar 2016 (v4)

HAL is a multi-disciplinary open access archive for the deposit and dissemination of scientific research documents, whether they are published or not. The documents may come from teaching and research institutions in France or abroad, or from public or private research centers.
L'archive ouverte pluridisciplinaire HAL, est destinée au dépôt et à la diffusion de documents scientifiques de niveau recherche, publiés ou non, émanant des établissements d'enseignement et de recherche français ou étrangers, des laboratoires publics ou privés. 


\title{
Study of a numerical scheme for miscible two-phase flow in porous media
}

\author{
R. Eymard*and V. Schleper ${ }^{\dagger}$
}

July 10, 2013

\begin{abstract}
We study the convergence of a finite volume scheme for a model of miscible two-phase flow in porous media. In this model, one phase can dissolve into the other one. The convergence of the scheme is proved thanks to an estimate on the two pressures, which allows to prove some estimates on the discrete time derivative of some nonlinear functions of the unknowns. Monotony arguments allow to show some properties on the limits of these functions. A key point in the scheme is to use particular averaging formula for the dissolution function arising in the space term.
\end{abstract}

KeYwords. Two-phase flow in porous medium, dissolution, finite volume methods, convergence study.

AMS CLASSIFICATION. 65N08, 65N12.

\section{Introduction}

This paper is focused on the study of the convergence of a numerical method for the approximation of two-phase flow in porous media, where dissolution of the gaseous phase can occur. Such a problem arises in different engineering frameworks. In the framework of hydrology, the water phase flow together with the air gaseous phase in the underground. In the framework of nuclear waste management, some gaseous hydrogen, produced by acid attack of metallic containers containing the nuclear waste, may flow within porous soils initially saturated with water. Let us first give the continuous model, which also holds for $\mathrm{CO} 2$ storage, gas production, and other situations. For $x \in \Omega$ and $t \in[0, T]$, we consider the functions $p_{w}, p_{g}$, weak solution in a sense precised below, to the following model, which describes the mass conservation of two species, a liquid and a gaseous ones. These equations can be written by

$$
\begin{array}{r}
\partial_{t} A_{w}^{w}+\operatorname{div} F_{w}^{w}=f_{w}, \\
\partial_{t}\left(A_{g}^{w}+A_{g}^{g}\right)+\operatorname{div}\left(F_{g}^{w}+F_{g}^{g}\right)=f_{g},
\end{array}
$$

where, for $m=w, g$ and $\ell=w, g$, the expression $A_{m}^{\ell}$ represents the mass of constituent $m$ in phase $\ell$ per unit volume, $F_{m}^{\ell}$ is the massic flux of constituent $m$ within phase $\ell$, and $f_{m}$

\footnotetext{
${ }^{*}$ LAMA UMR 8050, Université Paris-Est, France

${ }^{\dagger}$ corresponding author, Institut für Angewandte Analysis und Numerische Simulation, Universität Stuttgart, Germany
} 
is the massic source term of constituent $m$ per unit volume. In this model, the water phase $\ell=w$ may include the constituents $m=w$ (water) and $m=g$ (gas), whereas the gaseous phase $\ell=g$ may only contain the gas constituent $m=g$. Since we consider a flow across a porous medium, we may write

$$
\begin{aligned}
& A_{w}^{w}=\Phi(x) S(q) \rho_{w}, \quad F_{w}^{w}=-\Lambda \rho_{w} k_{w}(S(q)) \nabla p_{w}, \quad q=p_{w}-p_{g} \\
& A_{g}^{g}=\Phi(x) S(q) \rho_{w} X\left(p_{g}\right), \quad A_{g}^{g}=\Phi(x)(1-S(q)) \rho_{w} X\left(p_{g}\right), \\
& F_{g}^{w}=-\Lambda \rho_{w} k_{w}(S(q)) X\left(p_{g}\right) \nabla p_{w}-D \rho_{w} S(q) \nabla X\left(p_{g}\right), \quad F_{g}^{g}=-\Lambda \rho_{g} k_{g}(S(q)) \nabla p_{g},
\end{aligned}
$$

where $p_{g}$ and $p_{w}$ denote the gas and water pressure respectively and $\Lambda$ is the constant mobility coefficient. In the following, we assume $\Lambda=1$ to simplify the notation. The difference between the two pressures, $q$, is called the capillary pressure. $\rho_{g / w}$ are the (constant) mass densities of the two fluid phases, $\Phi(x)$ the porosity of the medium, defined as the volume occupied by the two fluid phases per unit volume and $D$ the diffusivity coefficient of the dissolved gas phase in the liquid phase, dedicated to the modeling of Fick's law (note that the part of the molecular diffusion in the coefficient $D$ is small compared to the effect of dispersed velocities at the pore scale). The function $S(q)$ denotes the water saturation defined as the volume occupied by the water phase per unit fluid volume (see Figure 1). The positive functions $k_{g / w}$, depending on the saturation, are the mobilities of the gas and water phase respectively (including the absolute permeability and the viscosity of the phase), involved in the generalized Darcy's law. Furthermore, $X$ is the mass fraction of the gaseous component dissolved in the water phase (see Figure 2).

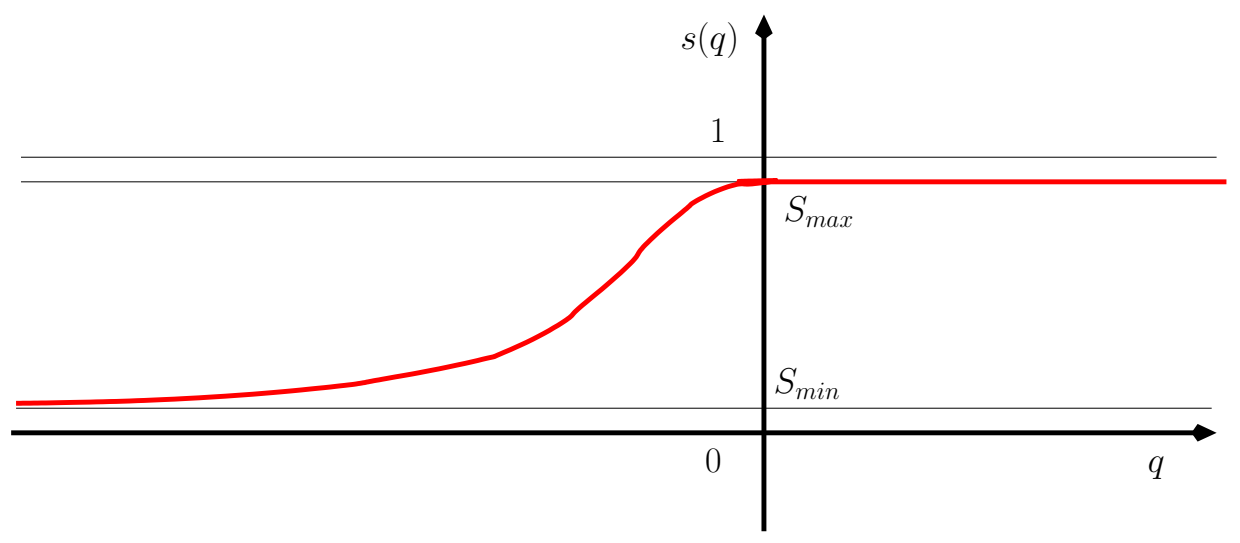

Figure 1: Function $S(q)$.

We then get the following resulting system of equations:

$$
\begin{aligned}
& q=p_{w}-p_{g}, \\
& \Phi(x) \partial_{t}\left(S(q) \rho_{w}\right)-\operatorname{div}\left(\rho_{w} k_{w}(S(q)) \nabla p_{w}\right)=f_{w}, \\
& \Phi(x) \partial_{t}\left(S(q) \rho_{w} X\left(p_{g}\right)+(1-S(q)) \rho_{g}\right) \\
& \quad-\operatorname{div}\left(\rho_{w} k_{w}(S(q)) X\left(p_{g}\right) \nabla p_{w}+\rho_{g} k_{g}(S(q)) \nabla p_{g}+D \rho_{w} S(q) \nabla X\left(p_{g}\right)\right)=f_{g},
\end{aligned}
$$

for which we consider the following boundary and initial conditions

$$
p_{g}(t, x)=0, \quad \quad p_{w}(t, x)=0 \quad \forall x \in \partial \Omega, t \in[0, T]
$$




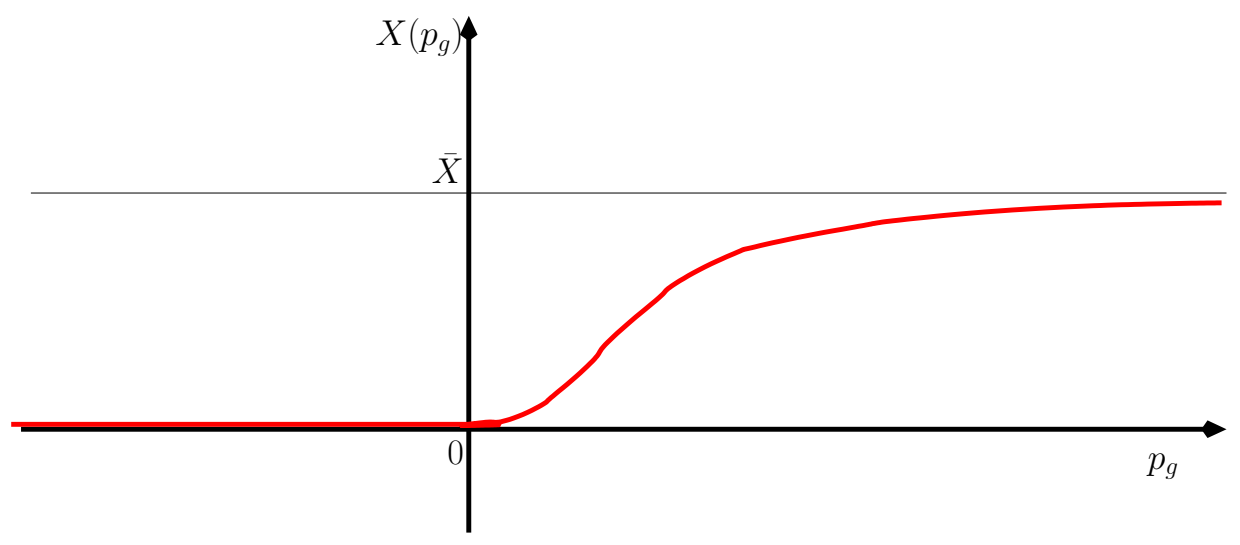

Figure 2: Function $X\left(p_{g}\right)$.

$$
p_{g}(x, 0)=p_{g}^{0}(x), \quad p_{w}(x, 0)=p_{w}^{0}(x) \quad \forall x \in \Omega .
$$

These data are assumed in this paper to satisfy the following hypotheses, all together denoted in the following by $\mathbf{( H )}$ :

$(\Omega, T) \Omega \subset \mathbb{R}^{d}$ is a polygonal domain (in an extended sense for $d=1,2,3$ ), $T \in(0,+\infty)$. We define $Q_{T}:=(0, T) \times \Omega$.

(Ini) $p_{w}^{0} \in \mathbf{L}^{2}(\Omega)$ and $p_{g}^{0} \in \mathbf{L}^{2}(\Omega)$.

(R) $\rho_{w}=$ const and $\rho_{g}=$ const.

(S) $S: \mathbb{R} \rightarrow\left[S_{\min }, S_{\max }\right]$ is a non-decreasing differentiable function with $0<$ $S_{\text {min }}<S_{\text {max }}<1$, such that $\sup _{\tau \in \mathbb{R}}\left|S^{\prime}(\tau)\right| \leq L_{S}$.

(X) $X: \mathbb{R} \rightarrow[0, \bar{X}]$ is a non-decreasing differentiable function with $X(0)=0$, such that $\sup _{\tau \in \mathbb{R}}\left|X^{\prime}(\tau)\right| \leq L_{X}$.

(D) $D \geq 0$ is a possibly null positive constant.

(K) $k_{w} \in \mathbf{C}^{\mathbf{0}, \mathbf{1}}([0,1])$ is a positive, non-decreasing function with $k_{w}^{\min }:=k_{w}\left(S_{\min }\right)>$ 0 and $k_{g} \in \mathbf{C}^{\mathbf{0}, \mathbf{1}}([0,1])$ is a positive, non-increasing function with $k_{g}^{\text {min }}:=$ $k_{g}\left(S_{\max }\right)>0$.

$(\Phi) \Phi: \Omega \rightarrow \mathbb{R}$ is a measurable function with $0<\Phi_{\min } \leq \Phi(x) \leq \Phi_{\max }$.

(f) $f_{i} \in \mathbf{L}^{2}\left(Q_{T}\right)$, for $i=w, g$ are given source terms.

Let us comment the hypotheses done here, with respect to the literature.

First considering the immiscible case (corresponding to set $\bar{X}=0$ in Hypothesis $(\mathbf{X})$ ), Problem (1.1) under Hypotheses (H) has been the subject of many theoretical and numerical studies.

Let us recall the reference book [11], where theoretical works have been done on the analysis of the continuous problem with $D=0$ in Hypothesis (D), and $S_{\min }=0$ and 
$S_{\max }=1$ instead of Hypothesis (S), dealing with degenerate parabolic equations. Let us also mention the theoretical study done in [5], showing the existence of a solution to the continuous problem in a case where the porous medium is nonrigid (modifying Hypothesis ( $\Phi)$ by introducing some dependence on the pressure).

Turning to convergence studies of numerical schemes in the immiscible case, many works are available in the literature, and it is not possible to cite all of them. If we restrict the literature to the finite volume schemes used in the industrial framework, let us note that the first convergence study of the phase-by-phase upstream weighting scheme has been done in [10], under modification of Hypothesis (S) (this study is done with $S_{\min }=0$ and $S_{\max }=$ 1 , and the function $S$ is replaced by its reciprocal function, hence defining the capillary pressure as a function of the saturation) and of $(\mathbf{K})$ with the addition of a series of technical hypotheses on the functions $k_{w}, k_{g}$. Let us then mention further works like [1] in the case of one compressible phase (modification of Hypothesis (R)) or [2], for the study of numerical schemes for two-phase flow with discontinuous capillary forces (modification of Hypothesis (S)).

Now turning to the literature in the miscible case, let us first cite [4] where the authors study the two-phase flow model, assuming compressibility (modification of Hypothesis $(\mathbf{R})$ ), diffusion (Hypothesis $(\mathbf{D})$ is changed in $D>0$ ) and mass exchange between the phases (with a mass exchange rate, instead of Hypothesis (X) stating the thermodynamical equilibrium between the phases), and they show the existence of a weak solution to this model. In [3], the authors show the existence of a weak solution for Problem (1.1), with $D>0$, but assuming the equilibrium Hypothesis $(\mathbf{X})$. Up to our knowledge, the present paper provides the first convergence study of a numerical scheme under Hypotheses $(\mathbf{H})$.

Let us emphasize that this study is the source of a series of difficulties. The problem posed by the first estimation is that it leads to consider nonlinear functions of the unknowns as test functions. Although this does not provide particular difficulties in the continuous case (thanks to Stampacchia's results), it prevents from using general schemes for the discretization of the space terms (like it is done in [9], concerning a wide class of discretization methods, namely the gradient schemes). On the contrary, we are led to use two-point flux approximation for the space terms, in the same spirit of [10] (and further works like [1] in the case of one compressible phase). Note that such a difficulty also arises in [7] or [2], or more generally in some elliptic problems with irregular data [6] where nonlinear test functions of the unknown must be used.

Let us mention that Hypothesis (S), which is an approximation of the physical situation, where $S_{\min }=0$ and $S_{\max }=1$, is done for enabling some estimates and convergence results (in the numerical tests, it is possible to take values for $\left(S_{\min }, S_{\max }\right)$ close to $\left.(0,1)\right)$. The question of relaxing this hypothesis by using the phase-by-phase upstream weighting scheme is open and seems to be quite difficult. Since our paper is focused on the difficulties due to the numerical approximation of the function $X$ in the space terms, we have preferred to use simpler arguments, deduced from Hypothesis (S). Let us also emphasize that we do not use $D>0$ for proving the convergence of the scheme, which means that the present analysis applies in the case where Problem (1.1) is a regularization of the immiscible two-phase flow problem by the addition of a dissolution term.

Remark 1.1 (Non-homogeneous Dirichlet boundary conditions and gravity terms). We could as well consider the case of non-homogeneous Dirichlet boundary conditions and the presence 
of gravity terms, which would only lead to additional terms in the estimates and in the convergence results.

Let us now provide the weak sense that is considered for a solution to Problem (1.1).

Definition 1.2 (Weak sense of a continuous solution). Under Hypotheses $(\mathbf{H})$, we say that $\left(p_{w}, p_{g}\right)$ is a weak solution to (1.1) if:

$$
\begin{gathered}
p_{g} \in \mathbf{L}^{\mathbf{2}}\left((0, T) ; \mathbf{H}_{\mathbf{0}}^{\mathbf{1}}(\Omega)\right), p_{w} \in \mathbf{L}^{\mathbf{2}}\left((0, T) ; \mathbf{H}_{\mathbf{0}}^{\mathbf{1}}(\Omega)\right) \text { and, for all } \varphi \in \mathbf{C}_{\mathbf{c}}^{\infty}(\Omega \times[0, T)) \\
\int_{0}^{T} \int_{\Omega}-\Phi(x) \rho_{w} S(q) \partial_{t} \varphi d x d t+\int_{0}^{T} \int_{\Omega} k_{w}(S(q)) \rho_{w} \nabla p_{w} \nabla \varphi d x d t \\
=\int_{\Omega} \Phi(x) \rho_{w} S\left(q^{0}\right) \varphi(x, 0) d x+\int_{0}^{T} \int_{\Omega} f_{w} \varphi d x d t \\
\int_{0}^{T} \int_{\Omega}-\Phi(x)\left(\rho_{w} S(q) X\left(p_{g}\right)+(1-S(q)) \rho_{g}\right) \partial_{t} \varphi d x d t \\
+\int_{0}^{T} \int_{\Omega}\left(k_{w}(S(q)) \rho_{w} X\left(p_{g}\right) \nabla p_{w}+k_{g}(S(q)) \rho_{g} \nabla p_{g}+D \rho_{w} S(q) \nabla X\left(p_{g}\right)\right) \nabla \varphi d x d t \\
\quad=\int_{\Omega} \Phi(x)\left(\rho_{w} S\left(q^{0}\right) X\left(p_{g}^{0}\right)-\left(1-S\left(q^{0}\right)\right) \rho-g\right) \varphi(x, 0) d x+\int_{0}^{T} \int_{\Omega} f_{g} \varphi d x d t,
\end{gathered}
$$

where $\mathbf{C}_{\mathbf{c}}^{\infty}(\Omega \times[0, T))$ denotes the set of the restrictions of all functions of $\mathbf{C}_{\mathbf{c}}^{\infty}(\Omega \times(-\infty, T))$ to $(\Omega \times[0, T))$.

This paper is organized as follows. In Section 2, we show briefly the continuous method used in order to prove the estimates, assuming sufficient regularity of the solution. This section details in particular the use of nonlinear test functions. Then, after presenting the discrete scheme in Section 3, we mimic the techniques used in section 2 to prove the estimates which hold in the discrete setting. From these estimates, we deduce the existence of at least one solution to the scheme and conclude the necessary compactness results, leading to the convergence proof of the scheme. In Section 4, simple numerical test cases show how some physical features are reproduced by this simple model. A short conclusion is finally given.

\section{Estimates in the continuous setting, assuming that the so- lution is regular}

In this section, we provide the computations which enable us to take into account the dissolution function $X$. For the sake of simplicity, we write the following estimates in the 1D case assuming $D=0$, which does not change the principles of the computations. The ideas and techniques applied in this section will then be carried over to the discrete setting in 3 .

Lemma 2.1. Let us assume that Hypotheses $(\mathbf{H})$ hold under the additional hypotheses that $d=1, f_{w}=f_{g}=0$ and that there exists a solution $\left(p_{w}, p_{g}\right)$ of Problem (1.2) which is sufficiently regular. Then, there exists a constant $C$ depending on the initial conditions $p_{g}^{0}$ and $p_{w}^{0}$ and on $k_{g}^{\min }, k_{w}^{\min }$ and $\Phi_{\min }$, such that for any solution of (1.2), we have

$$
\left\|\partial_{x} p_{g}\right\|_{\mathbf{L}^{2}\left(Q_{T}\right)} \leq C \quad \text { and } \quad\left\|\partial_{x} p_{w}\right\|_{\mathbf{L}^{2}\left(Q_{T}\right)} \leq C
$$


Proof. Recall that throughout this section, we have $D=0$ for simplicity. Now, we multiply equation (1.1b) by $\frac{p_{w}}{\rho_{w}}$ and equation (1.1c) by $\frac{p_{g}}{\rho_{g}}$ and add the obtained equations. Taking advantage of the fact that the mass densities $\rho_{g / w}$ are assumed to be constant in Hypothesis $(\mathbf{H})$, we obtain

$$
\begin{aligned}
& \Phi(x) \partial_{t}\left(S(q) \frac{\rho_{w}}{\rho_{g}} X+(1-S(q))\right) p_{g}+\Phi(x) \partial_{t}(S(q)) p_{w} \\
& \quad=\partial_{x}\left(\frac{\rho_{w}}{\rho_{g}} k_{w}(S(q)) X\left(p_{g}\right) \partial_{x} p_{w}+k_{g}(S(q)) \partial_{x} p_{g}\right) p_{g}+\partial_{x}\left(k_{w}(S(q)) \partial_{x} p_{w}\right) p_{w}
\end{aligned}
$$

To simplify the notations below, we define

$$
\tilde{S}(q):=\int_{0}^{q} S^{\prime}(\tau) \tau d \tau, \quad \tilde{X}(p):=\int_{0}^{p} X^{\prime}(\tau) \tau d \tau \quad \text { and } \quad \alpha=\frac{\rho_{w}}{\rho_{g}} .
$$

Then, the left hand side of (2.2) becomes

$$
\begin{aligned}
\Phi(x) & \partial_{t}\left(S(q) \alpha X\left(p_{g}\right)+(1-S(q))\right) p_{g}+\Phi(x) \partial_{t}(S(q)) p_{w} \\
& =\Phi(x) \partial_{t}(S(q)) q+\Phi(x) \alpha \partial_{t}\left(S(q) X\left(p_{g}\right)+1\right) p_{g} \\
& =\Phi(x)\left[\partial_{t}(\tilde{S}(q))+\alpha S \partial_{t}\left(X\left(p_{g}\right)\right) p_{g}+\alpha X\left(p_{g}\right) p_{g} \partial_{t}(S(q))\right] \\
& =\Phi(x)\left[\partial_{t}(\tilde{S}(q))+\partial_{t}\left(\alpha S(q) \tilde{X}\left(p_{g}\right)\right)\right]-\left[\alpha\left(\tilde{X}\left(p_{g}\right)-X\left(p_{g}\right) p_{g}\right) \partial_{x}\left(k_{w}(S(q)) \partial_{x} p_{w}\right)\right]
\end{aligned}
$$

and we can write $(2.2)$ as

$$
\begin{aligned}
\Phi(x) & {\left[\partial_{t}(\tilde{S}(q))+\partial_{t}\left(\alpha S(q) \tilde{X}\left(p_{g}\right)\right)\right] } \\
= & \partial_{x}\left(\alpha k_{w}(S(q)) X\left(p_{g}\right) \partial_{x} p_{w}+k_{g}(S(q)) \partial_{x} p_{g}\right) p_{g}+\partial_{x}\left(k_{w}(S(q)) \partial_{x} p_{w}\right) p_{w} \\
& +\alpha\left(\tilde{X}\left(p_{g}\right)-X\left(p_{g}\right) p_{g}\right) \partial_{x}\left(k_{w}(S(q)) \partial_{x} p_{w}\right)
\end{aligned}
$$

To obtain the desired estimates, we integrate (2.4) in space and time. Recall that $\tilde{X}(0)=0$, and $\frac{d}{d p}(X(p) p-\tilde{X}(p))=X(p)$, such that integration by parts yields

$$
\begin{aligned}
\int_{0}^{T} \int_{\Omega} & k_{w}(S(q))\left(\partial_{x} p_{w}\right)^{2} d x d t+\int_{0}^{T} \int_{\Omega} k_{g}(S(q))\left(\partial_{x} p_{g}\right)^{2} d x d t \\
& =\int_{\Omega} \Phi(x)\left[\left(\tilde{S}\left(q^{0}\right)-\tilde{S}(q(T))\right)+\left(\alpha S\left(q^{0}\right) \tilde{X}\left(p_{g}^{0}\right)-S(q(T)) \tilde{X}\left(p_{g}(T)\right)\right)\right] d x
\end{aligned}
$$

Now note that $S$ and $X$ are differentiable with uniformly bounded derivative by Hypothesis $(\mathbf{H})$. Furthermore we have $0<S(q)<1$, such that

$$
0 \leq \tilde{S}(q) \leq L_{S} \int_{0}^{q} \tau d \tau=\frac{L_{S}}{2} q^{2} \quad \text { and } \quad 0 \leq \alpha S(q) \tilde{X}\left(p_{g}\right) \leq \alpha \frac{L_{X}}{2} p_{g}^{2} .
$$

Note now, that $\alpha, D, S(q)$ and $X^{\prime}\left(p_{g}\right)$ are all non-negative by Hypothesis $(\mathbf{H})$. Furthermore, again by Hypothesis $(\mathbf{H})$, the functions $k_{w}$ and $k_{g}$ are bounded from below by a positive 
constant $k_{w}^{\text {min }}$ ( $k_{g}^{\text {min }}$ respectively), while $\Phi$ can be bounded from above by some positive constant $\Phi_{\max }$. Using (2.6) and the fact that $S, \tilde{S}$ and $\tilde{X}$ are positive, we have thus,

$$
\begin{aligned}
& k_{w}^{\min }\left\|\partial_{x} p_{w}\right\|_{\mathbf{L}^{2}\left(Q_{T}\right)}^{2}+k_{g}^{\min }\left\|\partial_{x} p_{g}\right\|_{\mathbf{L}^{2}\left(Q_{T}\right)}^{2} \\
& \leq \int_{0}^{T} \int_{\Omega} k_{w}(S(q))\left(\partial_{x} p_{w}\right)^{2} d x d t+\int_{0}^{T} \int_{\Omega} k_{g}(S(q))\left(\partial_{x} p_{g}\right)^{2} d x d t \\
& \leq \Phi_{\max } \frac{L_{S}}{2}\left\|q^{0}\right\|_{\mathbf{L}^{2}(\Omega)}^{2}+\Phi_{\max } \alpha \frac{L_{X}}{2}\left\|p_{g}^{0}\right\|_{\mathbf{L}^{2}(\Omega)}^{2}
\end{aligned}
$$

Next, we show that $\partial_{t} S(q)$ and $\partial_{t}\left(S(q) X\left(p_{g}\right)\right)$ are bounded in the dual norm. To this end, we define the weighted dual norm

$$
\|v\|_{\mathbf{H}_{\Phi}^{-1}(\Omega)}:=\sup \left\{\int_{\Omega} \Phi(x) v(x) \omega(x) d x \mid \omega \in \mathbf{H}_{\mathbf{0}}^{\mathbf{1}}(\Omega)(\Omega) \text { with }\|\omega\|_{\mathbf{H}_{\mathbf{0}}^{\mathbf{1}}(\Omega)(\Omega)}=1\right\} .
$$

Lemma 2.2. Let us assume that Hypotheses $(\mathbf{H})$ hold under the additional hypotheses that $d=1, f_{w}=f_{g}=0$ and that there exists a solution $\left(p_{w}, p_{g}\right)$ of Problem (1.2) which is sufficiently regular. Then, there exists a constant $C$ depending on the initial conditions $p_{g}^{0}$ and $p_{w}^{0}$ and on $k_{g}^{\min }, k_{w}^{\min }, k_{g}^{\max }, k_{w}^{\max }$ such that for any solution of (1.2), we have

$$
\left\|\partial_{t} S(q)\right\|_{\mathbf{L}^{\mathbf{2}}\left((0, T) ; \mathbf{H}_{\Phi}^{-1}(\Omega)\right)} \leq C \quad \text { and } \quad\left\|\partial_{t}\left(S(q) X\left(p_{g}\right)\right)\right\|_{\mathbf{L}^{\mathbf{2}\left((0, T) ; \mathbf{H}_{\Phi}^{-1}(\Omega)\right)}} \leq C
$$

Proof. Let $\omega \in \mathbf{H}_{\mathbf{0}}^{\mathbf{1}}(\Omega)(\Omega)$ be an arbitrary test function with $\|\omega\|_{\mathbf{H}_{\mathbf{0}}^{\mathbf{1}}(\Omega)(\Omega)}=1$. Using the definition of the weighted dual norm, we have

$$
\left\|\partial_{t} S(q)\right\|_{\mathbf{L}^{2}\left((0, T) ; \mathbf{H}_{\Phi}^{-1}(\Omega)\right)}=\int_{0}^{T}\left(\sup _{\substack{\omega \in \mathbf{H}_{\mathbf{0}}^{\mathbf{1}}(\Omega)(\Omega) \\\|\omega\|_{\mathbf{H}_{\mathbf{0}}^{\mathbf{1}}(\Omega)(\Omega)}=1}} \int_{\Omega} \Phi(x)\left(\partial_{t} S(q)\right) \omega d x\right)^{2} d t .
$$

To estimate this norm, note first that

$$
\begin{aligned}
\left(\int_{\Omega} \Phi(x)\left(\partial_{t} S(q)\right) \omega d x\right)^{2} & =\left(\int_{\Omega} k_{w}(S(q)) \partial_{x} p_{w} \partial_{x} \omega d x\right)^{2} \\
& \leq\left(k_{w}^{\max }\right)^{2} \int_{\Omega}\left(\partial_{x} p_{w}\right)^{2} d x\left\|\partial_{x} \omega\right\|_{\mathbf{L}^{2}(\Omega)}^{2}, \\
& \leq\left(k_{w}^{\max }\right)^{2}\left\|\partial_{x} p_{w}\right\|_{L^{2}(\Omega)}^{2}\|\omega\|_{\mathbf{H}_{\mathbf{0}}^{1}(\Omega)(\Omega)}^{2},
\end{aligned}
$$

where we used equation (1.1b) together with partial integration, the upper bound of the function $k_{w}$ and Hölder's inequality. Taking the maximum value over all $\omega \in \mathbf{H}_{\mathbf{0}}^{\mathbf{1}}(\Omega)(\Omega)$ with $\|\omega\|_{\mathbf{H}_{\mathbf{0}}^{\mathbf{1}}(\Omega)(\Omega)}=1$ and integrating in time yields

$$
\left\|\partial_{t} S(q)\right\|_{\mathbf{L}^{\mathbf{2}}\left((0, T) ; \mathbf{H}_{\Phi}^{-1}(\Omega)\right)}^{2}=\int_{0}^{T}\left(\sup _{\substack{\omega \in \mathbf{H}_{\mathbf{0}}^{\mathbf{1}}(\Omega)(\Omega) \\\|\omega\|_{\mathbf{H}_{\mathbf{0}}^{\mathbf{1}}(\Omega)(\Omega)}}} \int_{\Omega}\left(\Phi(x) \partial_{t} S(q)\right) \omega d x\right)^{2} d t
$$




$$
\begin{aligned}
& \leq\left(k_{w}^{\max }\right)^{2} \int_{0}^{T} \int_{\Omega}\left(\partial_{x} p_{w}\right)^{2} d x d t \cdot\left(\sup _{\substack{\omega \in \mathbf{H}_{\mathbf{0}}^{\mathbf{1}}(\Omega)(\Omega) \\
\|\omega\|_{\mathbf{H}_{\mathbf{0}}^{\mathbf{1}}(\Omega)(\Omega)}}}\|\omega\|_{\mathbf{H}_{\mathbf{0}}^{\mathbf{1}}(\Omega)(\Omega)}^{2}\right) \\
& \leq\left(k_{w}^{\max }\right)^{2}\left\|\partial_{x} p_{w}\right\|_{\mathbf{L}^{2}\left(Q_{T}\right)}^{2}
\end{aligned}
$$

Using the estimate of $\left\|\partial_{x} p_{w}\right\|_{\mathbf{L}^{\mathbf{2}}\left(Q_{T}\right)}$ provided by lemma 2.1 this proves the first estimate.

To prove the second estimate, we add equation (1.1b) and (1.1c) to obtain

$$
\Phi \partial_{t}(\alpha S X)=\partial_{x}\left((\alpha X+1) k_{w}(S) \partial_{x} p_{w}+k_{g}(S) \partial_{x} p_{g}\right)=0
$$

To estimate the dual norm $\left\|\partial_{t}\left(S(q) X\left(p_{g}\right)\right)\right\|_{\mathbf{L}^{2}\left((0, T) ; \mathbf{H}_{\Phi}^{-1}(\Omega)\right)}$, we proceed as above and start with and estimate of $\int_{\Omega} \Phi(x) \partial_{t}\left(S(q) X\left(p_{g}\right)\right) \omega d x$. Using (2.9) together with Hölder and Young inequalities, we have therefore

$$
\begin{aligned}
& \alpha^{2}\left(\int_{\Omega} \Phi \partial_{t}\left(S(q) X\left(p_{g}\right)\right) \omega d x\right)^{2}=\left(\int_{\Omega}(\alpha X+1) k_{w}(S) \partial_{x} p_{w} \partial_{x} \omega d x+\int_{\Omega} k_{g}(S) \partial_{x} p_{g} \partial_{x} \omega d x\right)^{2} \\
& \leq\|\omega\|_{\mathbf{H}_{\mathbf{0}}^{1}(\Omega)(\Omega)}^{2}\left((\alpha \bar{X}+1)^{2}\left(k_{w}^{\max }\right)^{2} \int_{\Omega}\left(\partial_{x} p_{w}\right)^{2} d x+\left(k_{g}^{\text {max }}\right)^{2} \int_{\Omega}\left(\partial_{x} p_{g}\right)^{2} d x\right. \\
& \left.\quad+(\alpha \bar{X}+1) k_{w}^{\text {max }} k_{g}^{\max }\left(\left(\int_{\Omega}\left|\partial_{x} p_{w} \partial_{x} \omega\right| d x\right)^{2}+\left(\int_{\Omega}\left|\partial_{x} p_{g} \partial_{x} \omega\right| d x\right)^{2}\right)\right) \\
& \leq\left((\alpha \bar{X}+1) k_{w}^{\text {max }}+k_{g}^{\text {max }}\right)\|\omega\|_{\mathbf{H}_{\mathbf{0}}^{1}(\Omega)(\Omega)}^{2}\left((\alpha \bar{X}+1)\left(k_{w}^{\text {max }}\right) \int_{\Omega}\left(\partial_{x} p_{w}\right)^{2} d x+\left(k_{g}^{\text {max }}\right) \int_{\Omega}\left(\partial_{x} p_{g}\right)^{2} d x\right)
\end{aligned}
$$

Taking the maximum value and integrating in time, we get

$$
\left\|\partial_{t}\left(S(q) X\left(p_{g}\right)\right)\right\|_{\mathbf{L}^{2}\left((0, T) ; \mathbf{H}_{\Phi}^{-1}(\Omega)\right)}^{2} \leq C_{1}\left\|\partial_{x} p_{w}\right\|_{\mathbf{L}^{2}\left(Q_{T}\right)}+C_{2}\left\|\partial_{x} p_{g}\right\|_{\mathbf{L}^{2}\left(Q_{T}\right)},
$$

where $C_{1}$ and $C_{2}$ only depend on $\alpha, \bar{X}, k_{w}$ and $k_{g}$.

\section{Finite volume scheme with two-point flux approximation}

We assume Hypotheses $(\mathbf{H})$, and consider a given mesh $\mathcal{T}$ with the following properties (see Figure 3) called Hypotheses (T) in the following.

(T) Denote by $\mathcal{E}$ the set of edges (in 2D) or sides (in 3D) respectively. Then,

(i) For every element $K \in \mathcal{T}$ there exists $\mathcal{E}_{K} \subset \mathcal{E}$ such that $\partial K=\bigcup_{\sigma \in \mathcal{E}_{K}} \bar{\sigma}$ and $\mathcal{E}=\bigcup_{K \in \mathcal{T}} \mathcal{E}_{K}$.

(ii) For $\sigma \in \mathcal{E}$ such that $\bar{K} \cap \bar{L}=\bar{\sigma} \neq \emptyset$ for some $K \in \mathcal{T}$ and $L \in \mathcal{T}$, we write also $K \mid L$ instead of $\sigma$.

(iii) The family of cell centers $\mathcal{P}:=\left(x_{K}\right)_{K \in \mathcal{T}}$ is such that $x_{K} \in \bar{K}$ for all $K \in \mathcal{T}$ and if $\sigma=K \mid L$, we have $x_{K} \neq x_{L}$ and the straight line $D_{K, L}$ connecting $x_{K}$ and $x_{L}$ is orthogonal to $K \mid L$. We denote by $n_{K \mid L}$ the normal vector of $\sigma$ directed into $L$. 
(iv) For any $\sigma \in \mathcal{E}$ with $\sigma \subset \partial \Omega$ and $\sigma \in \mathcal{E}_{K}$, we have $x_{K} \notin \sigma$ and the straight line through $x_{K}$ orthogonal to $\sigma$ is denoted by $D_{K \mid \sigma}$. We define $y_{\sigma}:=D_{K \mid \sigma} \cap \sigma$ and we denote by $n_{K \mid \sigma}$ the normal vector of $\sigma$ directed outside $\Omega$.

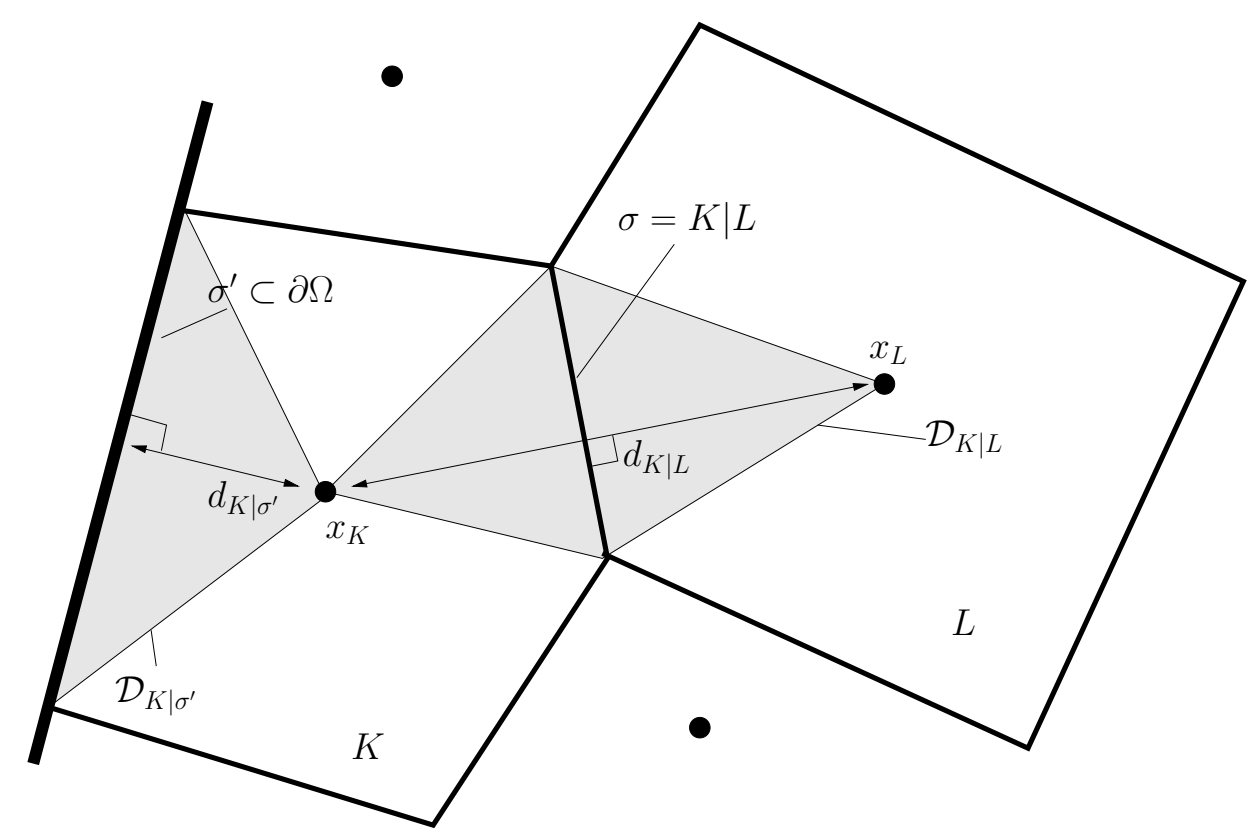

Figure 3: Notations for the discretization.

For simplicity, we introduce the following notation.

- The $(d-1)$-dimensional measure of $\sigma$ is denoted by $|\sigma|$.

- The set of interior edges is denoted by $\mathcal{E}_{i n t}$, the set of boundary edges by $\mathcal{E}_{\text {ext }}$.

- The euclidean distance of $x_{K}$ and $x_{L}$ for $L \in \mathcal{N}_{K}$ (with $\mathcal{N}_{K}$ the set of all neighbor elements) is denoted by $d_{\sigma}$ (or $d_{K \mid L}$ ). For $\sigma \in \mathcal{E}_{\text {ext }} \cap \mathcal{E}_{K}$, the euclidean distance between $x_{K}$ and $y_{\sigma}$ is denoted by $d_{K \mid \sigma}$.

- For $\sigma \in \mathcal{E}_{K} \cap \mathcal{E}_{\text {int }}$, we define $y_{\sigma}:=d_{K \mid L} \cap \sigma$ and denote by $d_{K \mid \sigma}$ the straight line connecting $x_{K}$ and $y_{\sigma}$.

- For $\sigma \in \mathcal{E}_{K} \cap \mathcal{E}_{\text {int }}$ we denote by $\mathcal{D}_{K \mid L}$ the diamond spanned by the diagonals $\sigma$ and $d_{K \mid L}$. For $\sigma \in \mathcal{E}_{K} \cap \mathcal{E}_{\text {ext }}$ we denote by $\mathcal{D}_{K \mid \sigma}$ the tetrahedron spanned by $\sigma$ and $D_{K \mid \sigma}$.

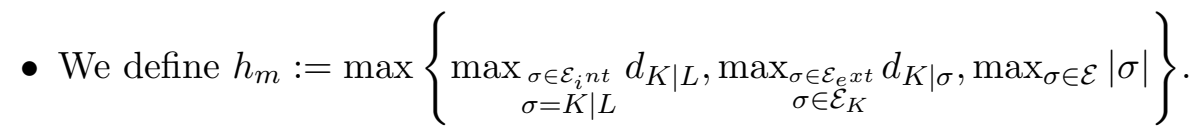

- We denote by $\mathcal{X}_{\mathcal{T}}$ the set of all functions $u: \Omega \rightarrow \mathbb{R}$ such that $u(x)=\sum_{K \in \mathcal{T}} u_{K} \chi_{K}(x)$ with $u_{K}=$ const and the characteristic function $\chi_{K}(x):=\left\{\begin{array}{ll}1, & x \in K \\ 0, & \text { else }\end{array}\right.$. 
For any sequence $t^{(0)}=0<t^{(1)} \ldots<t^{(N)}=T$, we denote the discrete time step by $\delta t^{\left(n+\frac{1}{2}\right)}=$ $t^{(n+1)}-t^{(n)}$ for $n=0, \ldots, N-1$. Furthermore, $h$ denotes the maximum value of the diameter of all $K \in \mathcal{T}$ and $\delta t^{\left(n+\frac{1}{2}\right)}, n=0, \ldots, N-1$. In the following definition, we introduce discrete bilinear forms and norms on the space $\mathcal{X}_{\mathcal{T}}$. Since the elements of $\mathcal{X}_{\mathcal{T}}$ are constant in each control volume and their gradient is a distribution whose support is the reunion of all edges of the mesh, the discrete version of an $H^{1}$ bilinear form and norm is obtained by summing, up to a geometric coefficient, the differences of the values across the edges.

Definition 3.1 (discrete bilinear forms and norm). For $u, v \in \mathcal{X}_{\mathcal{T}}$ and for a family $\left(w_{\sigma}\right)_{\sigma \in \mathcal{E}}$ of real values, we define the scalar product

$$
[u, v]_{w, \mathcal{T}}=\sum_{\substack{\sigma \in \mathcal{E}_{i n t} \\ \sigma=K \mid L}} w_{K \mid L} \frac{|\sigma|}{d_{K \mid L}}\left(u_{K}-u_{L}\right)\left(v_{K}-v_{L}\right)+\sum_{\substack{\sigma \in \mathcal{E}_{x x t} \\ \sigma \in \mathcal{E}_{K}}} w_{K \mid \sigma} \frac{|\sigma|}{d_{K \mid \sigma}} u_{K} v_{K},
$$

we define the following discrete operator

$$
\operatorname{div}_{K}(w, u)=\left[u, 1_{K}\right]_{w, \mathcal{T}}=\sum_{\substack{\sigma \in \mathcal{E}_{K} \cap \mathcal{E}_{i n t} \\ \sigma=K \mid L}} w_{K \mid L} \frac{|\sigma|}{d_{K \mid L}}\left(u_{K}-u_{L}\right)+\sum_{\substack{\sigma \in \mathcal{E}_{K} \cap \mathcal{E}-e x t \\ \sigma=K \mid \sigma}} w_{K \mid \sigma} \frac{|\sigma|}{d_{K \mid \sigma} \mid} u_{K}, \forall K \in \mathcal{T},
$$

and the following norm

$$
\|u\|_{\mathcal{T}}=\left([u, u]_{1, \mathcal{T}}\right)^{1 / 2}=\left(\sum_{\substack{\sigma \in \mathcal{E}_{\text {int }} \\ \sigma=K \mid L}} \frac{|\sigma|}{d_{K \mid L}}\left(u_{K}-u_{L}\right)^{2}+\sum_{\substack{\sigma \in \mathcal{E}_{\text {ext }} \\ \sigma \in \mathcal{E}_{K}}} w_{K \mid \sigma} \frac{|\sigma|}{d_{K \mid \sigma}} u_{K}^{2}\right)^{1 / 2} .
$$

Let $p_{g}^{(0)} \in \mathcal{X}_{\mathcal{T}}$ and $p_{w}^{(0)} \in \mathcal{X}_{\mathcal{T}}$ be given piecewise constant initial conditions. Then, we define the implicit scheme for a discretization of problem (1.2) by first setting

$$
\begin{array}{ll}
\Phi_{K}=\int_{K} \Phi(x) d x, & f_{c, K}^{(n+1)}=\frac{1}{\delta t^{\left(n+\frac{1}{2}\right)}} \int_{t^{(n)}}^{t^{(n+1)}} \int_{K} f_{c}(t, x) d x d t, c=w, g \\
p_{w}^{(n+1)} \in X_{\mathcal{T}}, p_{g}^{(n+1)} \in X_{\mathcal{T}}, & q^{(0)}=p_{w}^{(0)}-p_{g}^{(0)}, q^{(n+1)}=p_{w}^{(n+1)}-p_{g}^{(n+1)}, \\
S_{K}^{(0)}=S\left(q_{K}^{(0)}\right), S_{K}^{(n+1)}=S\left(q_{K}^{(n+1)}\right), & X_{K}^{(0)}=X\left(p_{g, K}^{(0)}\right), X_{K}^{(n+1)}=X\left(p_{g, K}^{(n+1)}\right), \\
\delta_{\mathcal{T}}^{\left(n+\frac{1}{2}\right)} S_{K}=\frac{S_{K}^{(n+1)}-S_{K}^{(n)}}{\delta t^{\left(n+\frac{1}{2}\right)}}, & \delta_{\mathcal{T}}^{\left(n+\frac{1}{2}\right)}\left(S_{K} X_{K}\right)=\frac{\left(S_{K}^{(n+1)} X_{K}^{(n+1)}\right)-\left(S_{K}^{(n)} X_{K}^{(n)}\right)}{\delta t^{\left(n+\frac{1}{2}\right)}} \\
\Psi_{K \mid \sigma}^{n+1} p_{g, K}^{n+1}=\int_{0}^{p_{g, K}^{(n+1)}} X(p) d p, & \Psi_{K \mid L}^{n+1}\left(p_{g, L}^{(n+1)}-p_{g, K}^{(n+1)}\right)=\int_{p_{g, K}^{(n+1)}}^{p_{g, L}^{(n+1)}} X(p) d p \\
k_{g, K \mid L}^{(0)}=k_{g}\left(S_{K \mid L}^{(0)}\right), k_{g, K \mid L}^{(n+1)}=k_{g}\left(S_{K \mid L}^{(n+1)}\right), & k_{w, K \mid L}^{(0)}=k_{w}\left(S_{K \mid L}^{(0)}\right), k_{w, K \mid L}^{(n+1)}=k_{w}\left(S_{K \mid L}^{(n+1)}\right) \\
k_{g, K \mid \sigma}^{(0)}=k_{g}\left(S_{K \mid \sigma}^{(0)}\right), k_{g, K \mid \sigma}^{(n+1)}=k_{g}\left(S_{K \mid \sigma}^{(n+1)}\right), & k_{w, K \mid \sigma}^{(0)}=k_{w}\left(S_{K \mid \sigma}^{(0)}\right), k_{w, K \mid \sigma}^{(n+1)}=k_{w}\left(S_{K \mid \sigma}^{(n+1)}\right) \\
S_{K \mid \sigma} \in\left[\min \left\{S_{K}, S(0)\right\}, \max \left\{S_{K}, S(0)\right\}\right], & S_{K \mid L}=S_{L \mid K} \in\left[\min \left\{S_{K}, S_{L}\right\}, \max \left\{S_{K}, S_{L}\right\}\right] \\
\forall K \in \mathcal{T}, \forall n \in\{0, \ldots, N-1\} . &
\end{array}
$$


and then compute a solution of

$$
\begin{aligned}
& \Phi_{K} \delta_{\mathcal{T}}^{\left(n+\frac{1}{2}\right)} S_{K}-\operatorname{div}_{K}\left(k_{w}^{(n+1)}, p_{w}^{(n+1)}\right)=f_{w, K}^{(n+1)} \\
& \Phi_{K} \alpha \delta_{\mathcal{T}}^{\left(n+\frac{1}{2}\right)}\left(S_{K} X_{K}\right)-\Phi_{K} \delta t^{\left(n+\frac{1}{2}\right)} S_{K}-\alpha \operatorname{div}_{K}\left(k_{w}^{(n+1)} \Psi^{(n+1)}, p_{w}^{(n+1)}\right) \\
& \quad-\operatorname{div}_{K}\left(k_{g}^{(n+1)}, p_{g}^{(n+1)}\right)-\alpha D \operatorname{div}_{K}\left(S_{\mathcal{E}}^{(n+1)}, X\left(p_{g}^{(n+1)}\right)\right)=f_{g, K}^{(n+1)}, \\
& \forall K \in \mathcal{T}, \forall n \in\{0, \ldots, N-1\} .
\end{aligned}
$$

Note that, since the function $X$ is monotonous, $\Psi$ fulfills

$$
\begin{aligned}
\Psi_{K \mid L} & =\frac{\int_{p_{g, K}^{(n+1)}}^{p_{g, L}^{(n+1)}} X(p) d p}{p_{g, L}-p_{g, K}} \in\left[\min \left\{X_{K}^{(n+1)}, X_{L}^{(n+1)}\right\}, \max \left\{X_{K}^{(n+1)}, X_{L}^{(n+1)}\right\}\right], \\
\Psi_{K \mid \sigma}= & \frac{\int_{0}^{p_{g, K}^{(n+1)}} X(p) d p}{p_{g, K}-0} \in\left[0, X_{K}^{(n+1)}\right]
\end{aligned}
$$

and is therefore a mean value at the cell boundary, especially chosen to fit the requirements of the algorithm. Let us also observe that the Dirichlet boundary conditions are taken into account through Definition 3.1 for the operator $\operatorname{div}_{K}(\cdot, \cdot)$, which involves finite differences with 0 at all the boundary edges.

To prove the convergence of the above defined algorithm, we proceed as follows. In Section 3.1, we prove, up to the extraction of subsequences, some compactness on a family of discrete solutions, and, in section 3.2, we show that the limit of a converging subsequence is a solution to (1.2).

\subsection{Existence of a discrete solution and discrete estimates}

In this Section, we prove estimates analogous to the ones in the continuous setting of section 2. These estimates will be used to prove some estimates on time and space translates of $S$ and $S X$ necessary in order to deduce the relative compactness of the sequences $S_{m}$ and $(S X)_{m}$ by the Kolmogorov-Riesz theorem.

Lemma 3.2. Let Hypotheses $(\mathbf{H})$ hold. Then there exists at least one solution to scheme (3.1) and a constant $C>0$ depending on the initial conditions $p_{g}^{(0)} \in \mathcal{X}_{\mathcal{T}}$ and $p_{w}^{(0)} \in \mathcal{X}_{\mathcal{T}}$ and on $\Omega$, $k_{g}^{\text {min }}, k_{w}^{\text {min }}, f_{w}, f_{g}, \alpha, \bar{X}$ and $\Phi$ such that for any solution of (3.1) we have

$$
\int_{0}^{T}\left\|p_{g}\right\|_{\mathcal{T}}^{2} d t \leq C \quad \int_{0}^{T}\left\|p_{w}\right\|_{\mathcal{T}}^{2} d t \leq C
$$

Proof. For any non-decreasing function $F$ satisfying $F(0)=0$ and any real value $s$, we define $\tilde{F}(s):=\int_{0}^{s} F^{\prime}(s) s d s$. Note that $\tilde{F}(s) \geq 0$ therefore,

$$
\tilde{F}(b)-\tilde{F}(a)=\int_{a}^{b} F^{\prime}(s) s d s=b(F(b)-F(a))-\int_{a}^{b}(F(s)-F(a)) d s \leq b(F(b)-F(a)) .
$$


Now, let us consider any family $r^{(n+1)} \in X_{\mathcal{T}}$, for $n=0, \ldots, N$, and compute $F_{\mathcal{T}}^{(n+1)}(x)=$ $\sum_{K \in \mathcal{T}} F\left(r_{K}^{(n+1)}\right) \chi_{K}(x)$. Using (3.3) with $b=r_{K}^{(n+1)}$ and $a=r_{K}^{(n)}$, we obtain

$$
\begin{aligned}
\sum_{n=0}^{N-1} \sum_{K \in \mathcal{T}}\left(F_{\mathcal{T}}^{(n+1)}(x)-F_{\mathcal{T}}^{(n)}(x)\right) r_{\mathcal{T}}^{(n+1)} & =\sum_{n=0}^{N-1} \sum_{K \in \mathcal{T}} \chi_{K}(x)\left(F\left(r_{K}^{(n+1)}\right)-F\left(r_{K}^{(n)}\right)\right) r_{K}^{(n+1)} \\
& \geq \sum_{n=0}^{N-1} \sum_{K \in \mathcal{T}} \chi_{K}(x)\left(\tilde{F}\left(r_{K}^{(n+1)}\right)-\tilde{F}\left(r_{K}^{(n)}\right)\right) \\
& \geq-\sum_{K \in \mathcal{T}} \chi_{K}(x) \tilde{F}\left(r_{K}^{0}\right) \\
& =-F_{\mathcal{T}}^{(0)}(x) .
\end{aligned}
$$

For any quantity with time index $(n+1)\left(\right.$ say $\left.Q^{(n+1)}\right)$ in $(3.1)$, we denote with the notation $Q$ (without the time index) the function of the time, equal to $Q^{(n+1)}$ in the interval $\left(t^{(n)}, t^{(n+1)}\right)$. Now, we multiply equation (3.1a) by $\delta t^{\left(n+\frac{1}{2}\right)} p_{w}^{(n+1)}$ and equation (3.1b) by $\omega=\delta t^{\left(n+\frac{1}{2}\right)} p_{g}^{(n+1)}$. Adding the two equations so obtained and summing on $K \in \mathcal{T}$ and on $n=0, \ldots, N-1$, we have

$$
\begin{aligned}
& \sum_{n=0}^{N-1} \sum_{K \in \mathcal{T}} \Phi_{K}\left(S_{K}^{(n+1)}-S_{K}^{(n)}\right) q_{K}^{(n+1)} \\
& +\sum_{n=0}^{N-1} \sum_{K \in \mathcal{T}} \alpha \Phi_{K}\left(S_{K}^{(n+1)} X_{K}^{(n+1)}-S_{K}^{(n)} X_{K}^{(n)}\right) p_{g, K}^{(n+1)} \\
& +\int_{0}^{T}\left[p_{w}, p_{w}\right]_{k_{w}, \mathcal{T}} d t \\
& +\int_{0}^{T}\left[p_{g}, p_{g}\right]_{k_{g}, \mathcal{T}} d t \\
& +\int_{0}^{T} \alpha\left[p_{w}, p_{g}\right]_{k_{w}} \Psi, \mathcal{T} d t \\
& +\int_{0}^{T} \alpha D\left[X\left(p_{g}\right), p_{g}\right]_{S_{\mathcal{E}}, \mathcal{T}} d t \\
& =\int_{0}^{T} \sum_{K \in \mathcal{T}}\left(f_{w, K} p_{w, K}+f_{g, K} p_{g, K}\right) d t .
\end{aligned}
$$

To obtain the desired estimates, we observe that (3.5f) is non-negative and that the terms in $(3.5 \mathrm{c})$ ans $(3.5 \mathrm{~d})$ can be bounded from above by

$$
\int_{0}^{T}\left(\left[p_{w}, p_{w}\right]_{k_{w}, \mathcal{T}}+\left[p_{g}, p_{g}\right]_{k_{g}, \mathcal{T}}\right) d t \geq k_{w}^{\min } \int_{0}^{T}\left\|p_{w}\right\|_{\mathcal{T}}^{2} d t+k_{g}^{\min } \int_{0}^{T}\left\|p_{g}\right\|_{\mathcal{T}}^{2} d t
$$

Thanks to (3.4) and (2.6), the term (3.5a) becomes

$$
\sum_{n=0}^{N-1} \sum_{K \in \mathcal{T}} \Phi_{K}\left(S_{K}^{(n+1)}-S_{K}^{(n)}\right) q_{K}^{(n+1)} \geq-\frac{L_{S}}{2} \sum_{K \in \mathcal{T}} \Phi_{K}\left(q_{K}^{(0)}\right)^{2}
$$




$$
\geq-\frac{\Phi_{\max } L_{S}}{2}\left\|q_{\mathcal{T}}^{0}\right\|_{L^{2}(\Omega)}^{2}
$$

Using again (3.4) and (2.6), the term (3.5b) can be written as

$$
\begin{aligned}
& \sum_{n=0}^{N-1} \sum_{K \in \mathcal{T}} \Phi_{K} \alpha\left(S_{K}^{(n+1)} X_{K}^{(n+1)}-S_{K}^{(n)} X_{K}^{(n)}\right) p_{g, K}^{(n+1)} \\
& \geq \sum_{n=0}^{N-1} \sum_{K \in \mathcal{T}} \Phi_{K} \alpha\left(S_{\mathcal{D}}^{(n)}\left(\tilde{X}_{K}^{(n+1)}-\tilde{X}_{K}^{(n)}\right)+X_{K}^{(n+1)} p_{g, K}^{(n+1)}\left(S_{K}^{(n+1)}-S_{K}^{(n)}\right)\right) d x \\
& =\sum_{n=0}^{N-1} \sum_{K \in \mathcal{T}} \Phi_{K} \alpha\left(\left(S_{K}^{(n+1)} \tilde{X}_{K}^{(n+1)}-S_{K}^{(n)} \tilde{X}_{K}^{(n)}\right)+\left(X_{K}^{(n+1)} p_{g, K}^{(n+1)}-\tilde{X}_{K}^{(n+1)}\right)\left(S_{K}^{(n+1)}-S_{K}^{(n)}\right)\right) \\
& \geq-\sum_{K \in \mathcal{T}} \Phi_{K} \alpha S_{K}^{(0)} \tilde{X}_{K}^{(0)}+\int_{0}^{T} \sum_{K \in \mathcal{T}} \alpha \hat{X}_{K} f_{w, K} d t \\
& \quad-\alpha \int_{0}^{T}\left[p_{w}, \hat{X}\right]_{k_{w}, \mathcal{T}} d t
\end{aligned}
$$

where $\hat{X}_{K}=X_{K} p_{g, K}-\tilde{X}_{K}=\int_{0}^{p_{g, K}} X(p) d p$. Note that $\left|\hat{X}_{K}\right| \leq \bar{X}\left|p_{g, K}\right|$. We then remark that, by definition of $\Psi_{K \mid L}$,

$$
\left[p_{w}, \hat{X}\right]_{k_{w}, \mathcal{T}}=\left[p_{w}, p_{g}\right]_{k_{w} \Psi, \mathcal{T}}
$$

term which vanishes when combining with $(3.5 \mathrm{e})$. We finally turn to a bound of the term arising in $(3.5 \mathrm{~g})$, combined with the last but one term issued from (3.6). We easily get, thanks to the Young inequality and to the discrete Poincaré inequality [8, Lemma 9.1 p.765], that

$$
\begin{aligned}
\int_{0}^{T} \sum_{K \in \mathcal{T}}\left(f_{w, K}\left(p_{w, K}-\alpha \hat{X}_{K}\right)+f_{g, K} p_{g, K}\right) d t & \\
\leq & \frac{\operatorname{diam}(\Omega)}{2}\left(\frac{\left\|f_{w}\right\|_{\mathbf{L}^{2}\left(Q_{T}\right)}^{2}}{k_{w}^{\text {min }}}+\frac{\left\|\left|f_{g}\right|+\alpha \bar{X}\left|f_{w}\right|\right\|_{\mathbf{L}^{2}\left(Q_{T}\right)}^{2}}{k_{g}^{\text {min }}}\right) \\
& \quad+\frac{1}{2}\left(k_{w}^{\text {min }} \int_{0}^{T}\left\|p_{w}\right\|_{\mathcal{T}}^{2} d t+k_{g}^{\text {min }} \int_{0}^{T}\left\|p_{g}\right\|_{\mathcal{T}}^{2} d t\right) .
\end{aligned}
$$

Combining all results and the fact that the term in (3.5f) is non-negative, we have

$$
\begin{aligned}
& \frac{1}{2}\left(k_{g}^{\min } \int_{0}^{T}\left\|p_{g}\right\|_{\mathcal{T}}^{2} d t+k_{w}^{m i n} \int_{0}^{T}\left\|p_{w}\right\|_{\mathcal{T}}^{2} d t\right) \\
& \quad \leq \Phi_{\max }\left(\alpha \frac{L_{X}}{2}\left\|p_{g, \mathcal{T}}^{(0)}\right\|_{L^{2}(\Omega)}+\frac{L_{S}}{2}\left\|q_{\mathcal{T}}^{0}\right\|_{L^{2}(\Omega)}^{2}\right)+C_{1}\left(\left\|f_{w}\right\|_{\mathbf{L}^{2}\left(Q_{T}\right)}^{2}+\left\|f_{g}\right\|_{\mathbf{L}^{2}\left(Q_{T}\right)}^{2}\right)
\end{aligned}
$$

where $C_{1}$ only depends on the data listed in the statement of the lemma, which concludes the proof of the estimates.

To deduce the existence of at least one solution, we substitute the functions $S$ and $X$ in (3.1) by $S_{\lambda}=\lambda S+(1-\lambda)$ and $X_{\lambda}=\lambda X+(1-\lambda)$. Note that the estimate (3.7) also holds 
for solutions of the modified scheme. Furthermore, the modified scheme has a solution for $\lambda=0$ (that is $p_{w}=0$ and $p_{g}=0$ ), such that we can deduce by a classical topological degree argument the existence of at least one solution for $\lambda=1$ and thus for (3.1).

We now define the operator $\delta_{\mathcal{T}}$ (without time index) by

$$
\delta_{\mathcal{T}} r_{\mathcal{T}}(t, x)=\delta_{\mathcal{T}}^{\left(n+\frac{1}{2}\right)} r_{K}=\frac{r_{K}^{(n+1)}-r_{K}^{(n)}}{\delta t^{\left(n+\frac{1}{2}\right)}}, x \in K, t \in\left(t^{(n)}, t^{(n+1)}\right), r^{(n)} \in X_{\mathcal{T}}, n=0, \ldots, N .
$$

To show the estimates on $\delta_{\mathcal{T}} S_{\mathcal{T}}$ and $\delta_{\mathcal{T}}\left(S_{\mathcal{T}} X_{\mathcal{T}}\right)$, we define the following dual norm.

Definition 3.3. Let $\mathcal{T}$ be a mesh on $\Omega$ that satisfies $(\mathbf{T})$. Then, we define the discrete dual semi-norm on $\mathbf{L}^{2}(\Omega)$ by

$$
|w|_{*, \mathcal{T}}:=\sup \left\{\sum_{K \in \mathcal{T}} \Phi_{K} w_{K} v_{K} \mid v \in \mathcal{X}_{\mathcal{T}} \text { with }\|v\|_{\mathcal{T}}=1\right\}, \quad \forall w \in \mathbf{L}^{\mathbf{2}}(\Omega) .
$$

Lemma 3.4. Let Hypotheses $(\mathbf{H})$ hold. Then, there exists a constant $C$ depending on the initial conditions $p_{g, \mathcal{T}}^{0}$ and $p_{w, \mathcal{T}}^{0}$, on $f_{w}, f_{g}, k_{w}, k_{g}$ and on $T$ such that for any solution of (3.1), we have

$$
\int_{0}^{T}\left|\delta_{\mathcal{T}} S_{\mathcal{T}}(., t)\right|_{*, \mathcal{T}}^{2} d t \leq C \quad \text { and } \quad \int_{0}^{T}\left|\delta_{\mathcal{T}}\left(S_{\mathcal{T}} X_{\mathcal{T}}\right)(., t)\right|_{*, \mathcal{T}}^{2} d t \leq C
$$

Proof. Let $\omega \in \mathcal{X}_{\mathcal{T}}$. From (3.1a), we get

$$
\begin{aligned}
& \left|\delta_{\mathcal{T}}^{\left(n+\frac{1}{2}\right)} S_{\mathcal{T}}\right|_{*, \mathcal{T}}^{2}=\left(\sup _{\substack{\omega \in \mathcal{X}_{\mathcal{T}} \\
\|\omega\|_{\mathcal{T}}=1}} \sum_{K \in \mathcal{T}} \Phi_{K} \delta_{\mathcal{T}}^{\left(n+\frac{1}{2}\right)} S_{K} \omega_{K}\right)^{2} \\
& =\left(\sup _{\substack{\omega \in \mathcal{X}_{\mathcal{T}} \\
\|\omega\|_{\mathcal{T}}=1}}\left(\left[p_{w}^{(n+1)}, \omega\right]_{k_{w}^{(n+1)}, \mathcal{T}}+\sum_{K \in \mathcal{T}} f_{w, K}^{(n+1)} \omega_{K}\right)\right)^{2} \\
& \leq\left(k_{w}^{\max }\right)^{2}\left\|p_{w}^{(n+1)}\right\|_{\mathcal{T}}^{2}+\operatorname{diam}(\Omega)\left\|f_{w}^{(n+1)}\right\|_{\mathbf{L}^{2}(\Omega)}^{2},
\end{aligned}
$$

by the Cauchy-Schwarz inequality, the boundedness of $k_{w}$ from above and the discrete Poincaré inequality. Multiplying by $\delta t^{\left(n+\frac{1}{2}\right)}$, and summing with respect to $n$, we get

$$
\int_{0}^{T}\left|\delta_{\mathcal{T}} S_{\mathcal{T}}(\cdot, t)\right|_{*, \mathcal{T}}^{2} d t \leq C
$$

To show the second estimate, we add equations (3.1a) and (3.1b) and follow the same steps as above to obtain

$$
\begin{aligned}
& \left|\delta_{\mathcal{T}}^{\left(n+\frac{1}{2}\right)}\left(S_{\mathcal{T}} X_{\mathcal{T}}\right)\right|_{*, \mathcal{T}}^{2} \\
& \quad \leq\left(\left(k_{w}^{\max }\right)^{2}(\alpha \bar{X}+1)^{2}\left\|p_{w}\right\|_{\mathcal{T}}^{2}+\left(\left(k_{g}^{\text {max }}\right)^{2}+(\alpha D \bar{X})^{2}\right)\left\|p_{g}\right\|_{\mathcal{T}}^{2}+\operatorname{diam}(\Omega)\left\|f_{g}^{(n+1)}\right\|_{\mathbf{L}^{2}(\Omega)}^{2}\right) .
\end{aligned}
$$

As for the first estimate, multiplication by $\delta t^{\left(n+\frac{1}{2}\right)}$, summation on $n$ and application of (3.7) yields the second estimate. 


\subsection{Convergence of the scheme}

In this section, we prove any limit of the numerical scheme is a solution to (1.2).

Lemma 3.5. Let Hypotheses $(\mathbf{H})$ hold. Let $\mathcal{T}$ be a space discretization satisfying Hypothesis (T). Denote, for any $p \in X_{\mathcal{T}}$, by

$$
\nabla_{\mathcal{T}} p:=d\left(\sum_{\substack{\sigma \in \mathcal{E}_{i n t} \\ \sigma=K \mid L}} \frac{p_{L}-p_{K}}{d_{K \mid L}} n_{K \mid L} \chi_{\mathcal{D}_{K \mid L}}+\sum_{\substack{\sigma \in \mathcal{E}_{\text {ext }} \\ \sigma \in \mathcal{E}_{K}}} \frac{0-p_{K}}{d_{K \mid \sigma}} n_{K \mid \sigma} \chi_{\mathcal{D}_{K \mid \sigma}}\right)
$$

the discrete gradient of $p$ associated to the mesh $\mathcal{T}$.

Let $\mathcal{T}_{m}$ be a sequence of space-time discretizations satisfying Hypothesis $(\mathbf{T})$ with $h_{m} \rightarrow 0$ as $m \rightarrow \infty$, and let $p_{m}^{(n)} \in X_{\mathcal{T}_{m}}, n=1, \ldots, N$ be such that the function $p_{m} \in \mathbf{L}^{2}\left(Q_{T}\right)$, defined by $p_{m}(\cdot, t)=p_{m}^{(n+1)}$ for $t \in\left(t^{(n)}, t^{(n+1)}\right)$ and extended by 0 outside $\Omega$, weakly converges as $m \rightarrow \infty$ in $\mathbf{L}^{2}\left(Q_{T}\right)$ to a function $p$, and such that $\int_{0}^{T}\left\|p_{m}(\cdot, t)\right\|_{\mathcal{T}_{m}}^{2} d t$ remains bounded.

Then the function $\nabla_{m} p_{m}$, defined by $\nabla_{m} p_{m}(\cdot, t)=\nabla_{\mathcal{T}_{m}} p_{m}^{(n+1)}$ for $t \in\left(t^{(n)}, t^{(n+1)}\right)$, also extended by 0 outside $\Omega$, converges weakly in $\mathbf{L}^{\mathbf{2}}\left((0, T) \times \mathbb{R}^{d}\right)^{d}$ to $\nabla p$ (hence proving that $p \in \mathbf{L}^{\mathbf{2}}\left((0, T) ; \mathbf{H}_{\mathbf{0}}^{\mathbf{1}}(\Omega)\right)$.

Proof. Let us first show the following property for a given space discretization $\mathcal{T}$ satisfying Hypothesis (T): for a given $\varphi \in\left(\mathbf{C}_{\mathbf{c}}^{\infty}\left(\mathbb{R}^{d}\right)\right)^{d}$ (not necessarily null at the boundary of $\Omega$ ) and $p \in X_{\mathcal{T}_{m}}$ be given, extended by 0 outside $\Omega$, then

$$
\left|\int_{\mathbb{R}^{d}}\left(\varphi \nabla_{\mathcal{T} p}+p \operatorname{div} \varphi\right) d x\right| \leq\|\varphi\|_{1, \infty} d|\Omega|\|p\|_{\mathcal{T}} h=\|\varphi\|_{1, \infty}|\Omega|\left\|\nabla_{\mathcal{T} p}\right\|_{\mathbf{L}^{2}(\Omega)} h .
$$

The equality in (3.11) immediately results from

$$
\begin{aligned}
\int_{\Omega}\left|\nabla_{\mathcal{T}} p(x)\right|^{2} d x & = \\
& \sum_{\substack{\sigma \in \mathcal{E}_{i n t} \\
\sigma=K \mid L}} \frac{|\sigma| d_{K \mid L}}{d} \frac{d^{2}\left(p_{L}-p_{K}\right)^{2}}{d_{K \mid L}}+\sum_{\substack{\sigma \in \mathcal{E}_{\text {ext }} \\
\sigma \in \mathcal{E}_{K}}} \frac{|\sigma| d_{K \mid \sigma}}{d} \frac{d^{2}\left(0-p_{K}\right)^{2}}{d_{K \mid \sigma}}=d\|p\|_{\mathcal{T}}^{2} .
\end{aligned}
$$

We denote by $\varphi_{K \mid L}=\frac{1}{\left|\mathcal{D}_{K \mid L}\right|} \int_{\mathcal{D}_{K \mid L}} \varphi d x$ the integral mean of $\varphi$ on the diamond $\mathcal{D}_{K \mid L}$. The quantity $\varphi_{K \mid \sigma}$ is defined analogously. Then we have

$$
\int_{\mathbb{R}^{d}}\left(\varphi \cdot \nabla_{\mathcal{T}} p+p \operatorname{div} \varphi\right) d x=\sum_{\sigma \in \mathcal{E}} \int_{\mathcal{D}_{\sigma}} \varphi \cdot \nabla_{\mathcal{T}} p d x+\sum_{K \in \mathcal{T}} p_{K} \int_{K} \operatorname{div} \varphi d x
$$

Using $\int_{\mathcal{D}_{\sigma}} \chi_{\mathcal{D}_{\sigma^{\prime}}}(x) d x=\frac{|\sigma| d_{\sigma}}{d}$ if $\sigma=\sigma^{\prime}$ and 0 otherwise, for the computation of the first term of the right hand side and using the Green formula for the second term of the right hand side, we obtain

$$
\int_{\mathbb{R}^{d}}\left(\varphi \cdot \nabla_{\mathcal{T} p}+p \operatorname{div} \varphi\right) d x
$$




$$
\begin{aligned}
= & \sum_{\substack{\sigma \in \mathcal{E}_{i n t} \\
\sigma=K \mid L}} d \frac{|\sigma| d_{K \mid L}}{d} \frac{p_{L}-p_{K}}{d_{K \mid L}} n_{K \mid L} \cdot \varphi_{K \mid L}+\sum_{\substack{\sigma \in \mathcal{E}_{\text {ext }} \\
\sigma \in \mathcal{E}_{K}}} d \frac{|\sigma| d_{K \mid \sigma}}{d} \frac{0-p_{K}}{d_{K \mid \sigma}} n_{K \mid \sigma} \cdot \varphi_{K \mid \sigma} \\
& \left.+\sum_{\substack{\sigma \in \mathcal{E}_{i n t} \\
\sigma=K \mid L}}\left(p_{K}-p_{L}\right) \int_{|\sigma|} \varphi \cdot n_{K \mid L} d s+\sum_{\substack{\sigma \in \mathcal{E}_{\text {ext }} \\
\sigma \in \mathcal{E}_{K}}} p_{K} \int_{|\sigma|} \varphi \cdot n_{K \mid \sigma} d s\right) \\
= & \sum_{\substack{\sigma \in \mathcal{E}_{i n t} \\
\sigma=K \mid L}}|\sigma|\left(p_{L}-p_{K}\right)\left(\frac{1}{\left|\mathcal{D}_{K \mid L}\right|} \int_{\left|\mathcal{D}_{K \mid L}\right|} \varphi_{K \mid L} \cdot n_{K \mid L} d x-\frac{1}{|\sigma|} \int_{|\sigma|} \varphi \cdot n_{K \mid L} d s\right) \\
& -\sum_{\substack{\sigma \in \mathcal{E}_{\text {ext }} \\
\sigma \in \mathcal{E}_{K}}}|\sigma| p_{K}\left(\frac{1}{\left|\mathcal{D}_{K|\sigma|}\right|} \int_{\left|\mathcal{D}_{K \mid \sigma}\right|} \varphi_{K \mid \sigma} \cdot n_{K \mid \sigma} d x-\frac{1}{|\sigma|} \int_{|\sigma|} \varphi \cdot n_{K \mid \sigma}\right) .
\end{aligned}
$$

Since $\varphi \in\left(\mathbf{C}_{\mathbf{c}}^{\infty}\left(\mathbb{R}^{d}\right)\right)^{d}$, we can bound

$$
\left|\frac{1}{\left|\mathcal{D}_{K \mid L}\right|} \int_{\left|\mathcal{D}_{K \mid L}\right|} \varphi_{K \mid L} \cdot n_{K \mid L} d x-\frac{1}{|\sigma|} \int_{|\sigma|} \varphi \cdot n_{K \mid L} d s\right| \leq\|\varphi\|_{1, \infty} h
$$

and

$$
\left|\frac{1}{\left|\mathcal{D}_{K|\sigma|}\right|} \int_{\left|\mathcal{D}_{K|\sigma|}\right|} \varphi_{K \mid \sigma} \cdot n_{K \mid \sigma} d x-\frac{1}{|\sigma|} \int_{|\sigma|} \varphi \cdot n_{K \mid \sigma}\right| \leq\|\varphi\|_{1, \infty} h .
$$

Thus, thanks to the Cauchy-Schwarz inequality,

$$
\begin{aligned}
& \left|\int_{\mathbb{R}^{d}}\left(\varphi \cdot \nabla_{\mathcal{T}} p+p \operatorname{div} \varphi\right) d x\right| \leq\|\varphi\|_{1, \infty} h\left(\sum_{\substack{\sigma \in \mathcal{E}_{\text {int }} \\
\sigma=K \mid L}}|\sigma|\left(\left|p_{L}-p_{K}\right|+\sum_{\substack{\sigma \in \mathcal{E}_{\text {ext }} \\
\sigma \in \mathcal{E}_{K}}}|\sigma|\left|p_{K}\right|\right)\right. \\
& \leq\|\varphi\|_{1, \infty} h\|p\|_{\mathcal{T}}\left(\sum_{\substack{\sigma \in \mathcal{E}_{\text {int }} \\
\sigma=K \mid L}}|\sigma| d_{K \mid L}+\sum_{\substack{\sigma \in \mathcal{E}_{\text {ext }} \\
\sigma \in \mathcal{E}_{K}}}|\sigma| d_{K \mid \sigma}\right) \\
& \leq\|\varphi\|_{1, \infty} d|\Omega|\|p\|_{\mathcal{T}} h,
\end{aligned}
$$

which completes the proof of (3.11). We can now prove the lemma. Thanks to (3.12), we deduce that, up to a subsequence, $\nabla_{m} p_{m}$ converges weakly in $\mathbf{L}^{2}\left((0, T) \times \mathbb{R}^{d}\right)^{d}$ to some function $G$. Integration in time of (3.11), using some regular time dependent function $\varphi$, yields

$$
\lim _{m \rightarrow \infty} \int_{0}^{T} \int_{\mathbb{R}^{d}}\left(\varphi \cdot \nabla_{m} p+p_{m} \operatorname{div} \varphi\right) d x d t=\int_{0}^{T} \int_{\mathbb{R}^{d}}(\varphi \cdot G+p \operatorname{div} \varphi) d x d t=0
$$


This shows that $G=\nabla p \in \mathbf{L}^{2}\left((0, T) \times \mathbb{R}^{d}\right)^{d}$ (which implies that the weak convergence of $\nabla_{m} p_{m}$ to $\nabla p$ holds for all the sequence), and therefore that $p \in \mathbf{L}^{\mathbf{2}}\left((0, T) ; \mathbf{H}^{\mathbf{1}}\left(\mathbb{R}^{d}\right)\right)$. Since $p=0$ almost everywhere outside $\Omega$, we get that $p \in \mathbf{L}^{\mathbf{2}}\left((0, T) ; \mathbf{H}_{\mathbf{0}}^{\mathbf{1}}(\Omega)\right)$.

Lemma 3.6. Let Hypotheses $(\mathbf{H})$ hold. Furthermore, let $\mathcal{T}_{m}$ be a sequence of space-time discretizations satisfying $(\mathbf{T})$ with $h_{m} \rightarrow 0$ as $m \rightarrow \infty$. We assume that $p_{g}^{(0)} \in \mathcal{X}_{\mathcal{T}}$ and $p_{w}^{(0)} \in \mathcal{X}_{\mathcal{T}}$ are given such that $p_{g}^{(0)} \in \mathbf{L}^{2}(\Omega)$ and $p_{w}^{(0)} \in \mathbf{L}^{2}(\Omega)$.

If $p_{w, \mathcal{T}_{m}}, p_{g, \mathcal{T}_{m}}, S_{\mathcal{T}_{m}}$ and $X_{\mathcal{T}_{m}}$ satisfy the scheme (3.1) for all $m \in \mathbb{N}$, the families $\left(S_{\mathcal{T}_{m}}\right)_{m \in \mathbb{N}}$ and $\left(S_{\mathcal{T}_{m}} X_{\mathcal{T}_{m}}\right)_{m \in \mathbb{N}}$ are relatively compact in $\mathbf{L}^{2}\left(Q_{T}\right)$.

Proof. The relative compactness of the family $\left(S_{\mathcal{T}_{m}}\right)_{m \in \mathbb{N}}$ can be shown exactly as in [9, Lemma 2.6]. To show the relative compactness of $\left(S_{\mathcal{T}_{m}} X_{\mathcal{T}_{m}}\right)_{m \in \mathbb{N}}$, we extend the functions $S_{\mathcal{T}_{m}}$ and $X_{\mathcal{T}_{m}}$ by 0 outside of $Q_{T}$. Recall that $S$ and $X$ are Lipschitz continuous and bounded from above. Let $\tau \in(0, T)$. For $t \in(0, T-\tau)$ we have then

$$
\begin{aligned}
& \int_{\Omega} \Phi(x)\left(\left(S_{\mathcal{T}_{m}} X_{\mathcal{T}_{m}}\right)(x, t+\tau)-\left(S_{\mathcal{T}_{m}} X_{\mathcal{T}_{m}}\right)(t, x)\right)^{2} d x \\
& \leq \bar{X} L_{S} \sum_{K \in \mathcal{T}_{m}} \Phi_{K}\left(\left(S_{K} X_{K}\right)(t+\tau)-\left(S_{K} X_{K}\right)(t)\right)\left(q_{K}(t+\tau)-q_{K}(t)\right) \\
& \quad+L_{X} \sum_{K \in \mathcal{T}_{m}} \Phi_{K}\left(\left(S_{K} X_{K}\right)(t+\tau)-\left(S_{K} X_{K}\right)(t)\right)\left(p_{g, K}(t+\tau)-p_{g, K}(t)\right)
\end{aligned}
$$

Using Definition 3.3, taking the square root and integrating in time, we obtain

$$
\begin{aligned}
\sqrt{\Phi_{\text {min }}} & \int_{0}^{T-\tau}\left\|\left(S_{\mathcal{T}_{m}} X_{\mathcal{T}_{m}}\right)(x, t+\tau)-\left(S_{\mathcal{T}_{m}} X_{\mathcal{T}_{m}}\right)(t, x)\right\|_{\mathbf{L}^{2}(\Omega)} d t \\
\leq & \sqrt{L_{S X}} \int_{0}^{T-\tau}\left|\left(S_{\mathcal{T}_{m}} X_{\mathcal{T}_{m}}\right)(x, t+\tau)-\left(S_{\mathcal{T}_{m}} X_{\mathcal{T}_{m}}\right)(t, x)\right|_{*, \mathcal{T}_{m}}^{\frac{1}{2}} \\
& \times\left(\left\|q_{m}(\cdot, t+\tau)-q_{m}(\cdot, t)\right\|_{\mathcal{T}_{m}}^{\frac{1}{2}}+\left\|p_{g, m}(\cdot, t+\tau)-p_{g, m}(\cdot, t)\right\|_{\mathcal{T}_{m}}^{\frac{1}{2}}\right) d t \\
\underset{\text { Young }}{\leq} & \frac{\sqrt{L_{S X}}}{\sqrt{\tau}} \int_{0}^{T-\tau}\left|\left(S_{\mathcal{T}_{m}} X_{\mathcal{T}_{m}}\right)(x, t+\tau)-\left(S_{\mathcal{T}_{m}} X_{\mathcal{T}_{m}}\right)(t, x)\right|_{*, \mathcal{T}_{m}} d t \\
& +\frac{\sqrt{L_{S X} \tau}}{2} \int_{0}^{T-\tau}\left\|q_{m}(\cdot, t+\tau)-q_{m}(\cdot, t)\right\|_{\mathcal{T}_{m}} d t \\
& +\frac{\sqrt{L_{S X} \tau}}{2} \int_{0}^{T-\tau}\left\|p_{g, m}(\cdot, t+\tau)-p_{g, m}(\cdot, t)\right\|_{\mathcal{T}_{m}} d t
\end{aligned}
$$

with $L_{S X}=\max \left\{\bar{X} L_{S}, L_{X}\right\}$. Now, we get by the definition of $q$

$$
\int_{0}^{T-\tau}\left\|p_{g, m}(\cdot, t+\tau)-p_{g, m}(\cdot, t)\right\|_{\mathcal{T}_{m}} d t \leq 2 \int_{0}^{T}\left\|p_{g, m}\right\|_{\mathcal{T}_{m}} d t \leq 2 \sqrt{T}\left(\int_{0}^{T}\left\|p_{g, m}\right\|_{\mathcal{T}_{m}}^{2} d t\right)^{\frac{1}{2}}
$$

and

$$
\int_{0}^{T-\tau}\left\|q_{m}(\cdot, t+\tau)-q_{m}(\cdot, t)\right\|_{\mathcal{T}_{m}} d t \leq 2 \int_{0}^{T}\left(\left\|p_{w, m}\right\|_{\mathcal{T}}+\left\|p_{g, m}\right\|_{\mathcal{T}}\right) d t
$$




$$
\leq 2 \sqrt{T}\left(\left(\int_{0}^{T}\left\|p_{w, m}\right\|_{\mathcal{T}_{m}}^{2} d t\right)^{\frac{1}{2}}+\left(\int_{0}^{T}\left\|p_{g, m}\right\|_{\mathcal{T}_{m}} d t\right)^{\frac{1}{2}}\right)
$$

Furthermore,

$$
\begin{array}{r}
\int_{0}^{T-\tau}\left|\left(S_{\mathcal{T}_{m}} X_{\mathcal{T}_{m}}\right)(x, t+\tau)-\left(S_{\mathcal{T}_{m}} X_{\mathcal{T}_{m}}\right)(t, x)\right|_{*, \mathcal{T}_{m}} d t \leq \tau \int_{0}^{T}\left|\delta_{\mathcal{T}}\left(S_{\mathcal{T}} X_{\mathcal{T}}\right)(\cdot, t)\right|_{*, \mathcal{T}} d t \\
\leq \tau \sqrt{T}\left(\int_{0}^{T}\left|\delta_{\mathcal{T}}\left(S_{\mathcal{T}} X_{\mathcal{T}}\right)(\cdot, t)\right|_{*, \mathcal{T}}^{2} d t\right)^{\frac{1}{2}}
\end{array}
$$

Gathering the results (3.13)-(3.15) and applying lemmas 3.2 and 3.4, we can conclude the existence of a constant $C$ such that for all $\tau \in \mathbb{R}$ the following estimate holds:

$$
\int_{\mathbb{R}}\left\|\left(S_{\mathcal{T}_{m}} X_{\mathcal{T}_{m}}\right)(x, t+\tau)-\left(S_{\mathcal{T}_{m}} X_{\mathcal{T}_{m}}\right)(t, x)\right\|_{\mathbf{L}^{2}(\Omega)} d t \leq T C \sqrt{|\tau|}
$$

It remains to estimate the space translates in $\mathbb{R}^{d}$ (recall that the functions are extended by 0 outside $\Omega$ ). For every $\xi \in \mathbb{R}^{d}$, we define the domain $\Omega^{\xi}:=\{x \in \Omega \mid x+\xi \in \Omega\}$. Then, the Lipschitz continuity of $S$ and $X$ yield

$$
\begin{aligned}
& \left\|\left(S_{\mathcal{T}_{m}} X_{\mathcal{T}_{m}}\right)(x+\xi, t)-\left(S_{\mathcal{T}_{m}} X_{\mathcal{T}_{m}}\right)(t, x)\right\|_{\mathbf{L}^{2}\left(\mathbb{R}^{d}\right)}^{2} \\
& \leq \tilde{C}|\xi|+\left\|\left(S_{\mathcal{T}_{m}} X_{\mathcal{T}_{m}}\right)(\cdot+\xi, t)-\left(S_{\mathcal{T}_{m}} X_{\mathcal{T}_{m}}\right)(\cdot, t)\right\|_{\mathbf{L}^{2}\left(\Omega^{\xi}\right)}^{2} \\
& \leq \tilde{C}|\xi|+L_{S}^{2} \bar{X}^{2}\left\|q_{\mathcal{T}_{m}}(\cdot+\xi, t)-q_{\mathcal{T}_{m}}(\cdot, t)\right\|_{\mathbf{L}^{2}(\Omega \xi)}^{2}+L_{X}^{2}\left\|p_{g, \mathcal{T}_{m}}(\cdot+\xi, t)-p_{g, \mathcal{T}_{m}}(\cdot, t)\right\|_{\mathbf{L}^{2}(\Omega \xi)}^{2} \\
& \leq \tilde{C}|\xi|+\bar{X}^{2} L_{S}^{2}\left\|p_{g, \mathcal{T}_{m}}(\cdot+\xi, t)-p_{g, \mathcal{T}_{m}}(\cdot, t)\right\|_{\mathbf{L}^{2}\left(\Omega^{\xi}\right)}^{2}+\left(\bar{X}^{2} L_{S}^{2}+L_{X}^{2}\right)\left\|p_{g, \mathcal{T}_{m}}(\cdot+\xi, t)-p_{g, \mathcal{T}_{m}}(\cdot, t)\right\|_{\mathbf{L}^{\mathbf{2}\left(\Omega^{\xi}\right)}}^{2}
\end{aligned}
$$

A classical result (see e.g. [8, Lemma 9.3]) yields

$$
\begin{aligned}
& \left\|p_{g, \mathcal{T}_{m}}(\cdot+\xi, t)-p_{g, \mathcal{T}_{m}}(\cdot, t)\right\|_{\mathbf{L}^{2}\left(\Omega^{\xi}\right)}^{2} \leq\left\|p_{g}\right\|_{\mathcal{T}_{m}}^{2}|\xi|(|\xi|+C \operatorname{size}(\mathcal{T})) \\
& \left\|p_{w, \mathcal{T}_{m}}(\cdot+\xi, t)-p_{w, \mathcal{T}_{m}}(\cdot, t)\right\|_{\mathbf{L}^{2}\left(\Omega^{\xi}\right)}^{2} \leq\left\|p_{w}\right\|_{\mathcal{T}_{m}}^{2}|\xi|(|\xi|+C \operatorname{size}(\mathcal{T})) .
\end{aligned}
$$

Integration in time and application of lemma 3.2 yields

$$
\int_{0}^{T}\left\|\left(S_{\mathcal{T}_{m}} X_{\mathcal{T}_{m}}\right)(x+\xi, t)-\left(S_{\mathcal{T}_{m}} X_{\mathcal{T}_{m}}\right)(t, x)\right\|_{\mathbf{L}^{2}\left(\mathbb{R}^{d}\right)}^{2} d t \leq T C|\xi|
$$

We can therefore conclude the relative compactness of the families $\left(S_{\mathcal{T}_{m}}\right)_{m \in \mathbb{N}}$ and $\left(S_{\mathcal{T}_{m}} X_{\mathcal{T}_{m}}\right)_{m \in \mathbb{N}}$ in $\mathbf{L}^{2}\left(Q_{T}\right)$ by the Kolmogorov-Riesz Theorem.

Let us state the following lemma, the proof of which is recalled in [9, lemma 2.7].

Lemma 3.7 (Minty's trick). Let $\Omega$ be an open bounded subset of $\mathbb{R}^{d}, d \geq 1, T>0, Q_{T}=$ $(0, T) \times \Omega$, and let $F: \mathbb{R} \rightarrow \mathbb{R}$ be a bounded continuous nondecreasing function. If there is a sequence $\left(u_{m}\right)_{m \in \mathbb{N}} \subset \mathbf{L}^{2}\left(Q_{T}\right)$ such that

1. there exists $u \in \mathbf{L}^{2}\left(Q_{T}\right)$ such that $\left(u_{m}\right)_{m \in \mathbb{N}}$ converges weakly to $u$ in $\mathbf{L}^{2}\left(Q_{T}\right)$, 
2. there exists a function $\widehat{F} \in \mathbf{L}^{2}\left(Q_{T}\right)$ such that $\left(F\left(u_{m}\right)\right)_{m \in \mathbb{N}}$ converges to $\widehat{F}$ in $\mathbf{L}^{2}\left(Q_{T}\right)$, then $\widehat{F}(t, x)=F(u(t, x))$ for a.e. $(t, x) \in Q_{T}$.

Lemma 3.8. Let Hypotheses $(\mathbf{K})$ hold and let $\mathcal{T}_{m}$ be a sequence of space-time discretizations satisfying $(\mathbf{T})$ with $h_{m} \rightarrow 0$ as $m \rightarrow \infty$. Let $q_{m}^{(n)} \in X_{\mathcal{T}_{m}}, n=1, \ldots, N$ be such that the function $q_{m} \in \mathbf{L}^{2}\left(Q_{T}\right)$, defined by $q_{m}(\cdot, t)=q_{m}^{(n+1)}$ for $t \in\left(t^{(n)}, t^{(n+1)}\right)$ is such that $\int_{0}^{T}\left\|q_{m}(\cdot, t)\right\|_{\mathcal{T}_{m}}^{2} d t$ remains bounded and $S\left(q_{m}\right)$ converges to some function $\widehat{S}$ as $m \rightarrow \infty$ in $\mathbf{L}^{2}\left(Q_{T}\right)$. Furthermore let us define the following piecewise constant functions on the diamonds (omitting some indices $m$ ):

$$
\begin{aligned}
K_{w, \mathcal{T}_{m}} & :=\sum_{\substack{\sigma \in \mathcal{E}_{i n t} \\
\sigma=K \mid L}} k_{w}\left(S_{K \mid L}\right) \chi_{\mathcal{D}_{K \mid L}}+\sum_{\substack{\sigma \in \mathcal{E}_{\text {ext }} \\
\sigma \in \mathcal{E}_{K}}} k_{w}\left(S_{K \mid \sigma}\right) \chi_{\mathcal{D}_{K \mid \sigma}} \\
K_{g, \mathcal{T}_{m}} & :=\sum_{\substack{\sigma \in \mathcal{E}_{i n t} \\
\sigma=K \mid L}} k_{g}\left(S_{K \mid L}\right) \chi_{\mathcal{D}_{K \mid L}}+\sum_{\substack{\sigma \in \mathcal{E}_{\text {ext }} \\
\sigma \in \mathcal{E}_{K}}} k_{g}\left(S_{K \mid \sigma}\right) \chi_{\mathcal{D}_{K \mid \sigma}},
\end{aligned}
$$

where, denoting by $S_{K}=S\left(q_{K}\right)$ for all $K \in \mathcal{T}_{m}$, we assume that the values $S_{K \mid \sigma}$ and $S_{K \mid L}$ only ensure $S_{K \mid \sigma} \in\left[\min \left\{S_{K}, S(0)\right\}, \max \left\{S_{K}, S(0)\right\}\right]$ and $S_{K \mid L}=S_{L \mid K} \in\left[\min \left\{S_{K}, S_{L}\right\}, \max \left\{S_{K}, S_{L}\right\}\right]$. Then, $\left(K_{w, \mathcal{T}_{m}}\right)_{m \in \mathbb{N}}$ converges to $k_{w}(\widehat{S})$ in $\mathbf{L}^{2}\left(Q_{T}\right)$ and $\left(K_{g, \mathcal{T}_{m}}\right)_{m \in \mathbb{N}}$ converges to $k_{g}(\widehat{S})$ in $\mathbf{L}^{2}\left(Q_{T}\right)$.

Proof. Denote by $L_{K_{w}}$ the Lipschitz constant of $k_{w}$ and recall that

$$
h_{m}:=\max \left\{\max _{\substack{\sigma \in \mathcal{E}_{i n t} t \\ \sigma=K \mid L}} d_{K \mid L}, \max _{\substack{\sigma \in \mathcal{E}_{e x t} \\ \sigma \in \mathcal{E}_{K}}} d_{K \mid \sigma}, \max _{\sigma \in \mathcal{E}}|\sigma|\right\} .
$$

Recall that $S_{K \mid L} \in\left[\min \left\{S_{K}, S_{L}\right\}, \max \left\{S_{K}, S_{L}\right\}\right]$ and $S_{K \mid \sigma} \in\left[S(0), S_{K}\right]$. We deduce therefore that $\left(S_{K \mid L}-S_{K}\right)^{2} \leq\left(S_{L}-S_{K}\right)^{2}$ and $\left(S_{K \mid \sigma}-S_{K}\right)^{2} \leq\left(S_{K}-S(0)\right)^{2}$ and obtain

$$
\begin{aligned}
& \int_{\Omega}\left(K_{w, \mathcal{T}_{m}}-k_{w}\left(S_{\mathcal{T}_{m}}\right)\right)^{2} d x \\
& =\sum_{K \in \mathcal{T}_{m}}\left(\sum_{\substack{\sigma \in \mathcal{E}_{K} \cap \mathcal{E}_{i n t} \\
\sigma=K \mid L}}\left(k_{w}\left(S_{K \mid L}\right)-k_{w}\left(S_{K}\right)\right)^{2} \frac{1}{d}|\sigma| d_{K, \sigma}+\sum_{\substack{\sigma \in \mathcal{E}_{K} \cap \mathcal{E}-e x t \\
\sigma=K \mid \sigma}}\left(k_{w}\left(S_{K \mid \sigma}\right)-k_{w}\left(S_{K}\right)\right)^{2} \frac{1}{d}|\sigma| d_{K, \sigma}\right) \\
& \leq L_{K_{w}} \frac{h_{m}}{d} \sum_{K \in \mathcal{T}_{m}}\left(\sum_{\substack{\sigma \in \mathcal{E}_{K} \cap \mathcal{E}_{i n t} \\
\sigma=K \mid L}} \frac{|\sigma|}{d_{K \mid L}}\left(S_{K \mid L}-S_{K}\right)^{2} d_{K \mid L}+\sum_{\substack{\sigma \in \mathcal{E}_{K} \cap \mathcal{E}-e x t \\
\sigma=K \mid \sigma}} \frac{|\sigma|}{d_{K \mid \sigma}}\left(S_{K \mid \sigma}-S_{K}\right)^{2} d_{K, \sigma}\right) \\
& \leq L_{K_{w}} \frac{h_{m}^{2}}{d} \sum_{K \in \mathcal{T}_{m}}\left(\sum_{\substack{\sigma \in \mathcal{E}_{K} \cap \mathcal{E}_{i n t} \\
\sigma=K \mid L}} \frac{|\sigma|}{d_{K \mid L}}\left(S_{L}-S_{K}\right)^{2}+\sum_{\substack{\sigma \in \mathcal{E}_{K} \cap \mathcal{E}-e x t \\
\sigma=K \mid \sigma}} \frac{|\sigma|}{d_{K \mid \sigma}}\left(S_{K}-S(0)\right)^{2}\right)
\end{aligned}
$$


$\leq L_{K_{w}} \frac{h_{m}^{2}}{d} L_{S}^{2}\left\|q_{m}\right\|_{\mathcal{T}_{m}}^{2}$

Integration in time and the bound on $\int_{0}^{T}\left\|q_{m}(\cdot, t)\right\|_{\mathcal{T}_{m}}^{2} d t$ yield

$$
\lim _{m \rightarrow \infty} \int_{0}^{T} \int_{\Omega}\left(K_{w, \mathcal{T}_{m}}-k_{w}\left(S_{m}\right)\right)^{2} d x d t=\lim _{m \rightarrow \infty} C h_{m}^{2}=0
$$

since $h_{m} \rightarrow 0$ for $m \rightarrow \infty$. Due to the regularity of $k_{w}$ and the strong convergence of $S_{m}$ the assertion follows. Analogously, we can prove the convergence of $K_{g}$ to $k_{g}(\widehat{S})$ in $\mathbf{L}^{2}\left(Q_{T}\right)$.

Lemma 3.9. Let Hypotheses $(\mathbf{K})$ hold and let $\mathcal{T}_{m}$ be a sequence of space-time discretizations satisfying $(\mathbf{T})$ with $h_{m} \rightarrow 0$ as $m \rightarrow \infty$. Let $p_{g, m}^{(n)} \in X_{\mathcal{T}_{m}}, n=1, \ldots, N$ be such that the function $p_{g, m} \in \mathbf{L}^{2}\left(Q_{T}\right)$, defined by $p_{g, m}(\cdot, t)=p_{g, m}^{(n+1)}$ for $t \in\left(t^{(n)}, t^{(n+1)}\right)$ is such that $\int_{0}^{T}\left\|p_{g, m}(\cdot, t)\right\|_{\mathcal{T}_{m}}^{2} d t$ remains bounded and $X\left(p_{g, m}\right)$ converges to some function $\widehat{X}$ as $m \rightarrow \infty$ in $\mathbf{L}^{\mathbf{2}}\left(Q_{T}\right)$. Furthermore define, for a.e. $t \in(0, T)$,

$$
\Psi_{K \mid \sigma}(t) p_{g, K}(t)=\int_{0}^{p_{g, K}(t)} X(p) d p, \quad \Psi_{K \mid L}(t)\left(p_{g, L}(t)-p_{g, K}(t)\right)=\int_{p_{g, K}(t)}^{p_{g, L}(t)} X(p) d p
$$

and

$$
\Psi_{\mathcal{T}_{m}}:=\sum_{\substack{\sigma \in \mathcal{E}_{\text {int }} \\ \sigma=K \mid L}} \Psi_{K \mid L} \chi_{\mathcal{D}_{K \mid L}}+\sum_{\substack{\sigma \in \mathcal{E}_{\text {ext }} \\ \sigma \in \mathcal{E}_{K}}} \Psi_{K \mid \sigma} \chi_{\mathcal{D}_{K \mid \sigma}} .
$$

Then, $\left(\Psi_{\mathcal{T}_{m}}\right)_{m \in \mathbb{N}}$ converges to $\widehat{X}$ in $\mathbf{L}^{2}\left(Q_{T}\right)$.

Proof. First, recall that $X$ is monotone and non-decreasing such that we get by definition of $\Psi_{K \mid L}$ :

$$
\begin{aligned}
\left(\Psi_{K \mid L}-X\left(p_{g, K}\right)\right)^{2} & =\left(\frac{\int_{p_{g, K}}^{p_{g, L}}\left(X(p)-X\left(p_{g, K}\right)\right) d p}{p_{g, L}-p_{g, K}}\right)^{2} \\
& \leq\left(\frac{\left(X\left(p_{g, L}\right)-X\left(p_{g, K}\right)\right)\left(p_{g, L}-p_{g, K}\right)}{p_{g, L}-p_{g, K}}\right)^{2} \\
& =\left(X\left(p_{g, L}\right)-X\left(p_{g, K}\right)\right)^{2}
\end{aligned}
$$

and analogously

$$
\left(\Psi_{K \mid \sigma}-X\left(p_{g, K}\right)\right)^{2}=\left(\frac{\int_{0}^{p_{g, K}}\left(X(p)-X\left(p_{g, K}\right)\right) d p}{p_{g, K}}\right)^{2} \leq\left(\frac{X\left(p_{g, K}\right) p_{g, K}}{p_{g, K}}\right)^{2}=X\left(p_{g, K}\right)^{2}
$$

Analogously to the proof of lemma 3.8 we get

$$
\int_{\Omega}\left(\Psi_{\mathcal{T}_{m}}-X\left(p_{g, \mathcal{T}_{m}}\right)\right)^{2} d x \leq \frac{h_{m}^{2}}{d} L_{X}\left\|p_{g, \mathcal{T}_{m}}\right\|_{\mathcal{T}_{m}} .
$$

Again, integration in time and the bound on $\int_{0}^{T}\left\|p_{g, m}(\cdot, t)\right\|_{\mathcal{T}_{m}}^{2} d t$ yield

$$
\lim _{m \rightarrow \infty}\left\|\Psi_{\mathcal{T}_{m}}-X\left(p_{g, \mathcal{T}_{m}}\right)\right\|_{\mathbf{L}^{\mathbf{2}}\left(Q_{T}\right)} \leq \lim _{m \rightarrow \infty} C h_{m}^{2}=0
$$

which concludes the proof. 
We may now state and prove the convergence result.

Theorem 3.10. [Convergence of the scheme] Let Hypotheses $(\mathbf{H})$ hold and let $\mathcal{T}_{m}$ be a sequence of space-time discretizations satisfying $(\mathbf{T})$ with $h_{m} \rightarrow 0$ as $m \rightarrow \infty$. Let $\left(p_{g, \mathcal{T}_{m}}^{(0)}\right)_{m \in \mathbb{N}}$ and $\left(p_{w, \mathcal{T}_{m}}^{(0)}\right)_{m \in \mathbb{N}}$ be a family of given initial conditions such that $\lim _{m \rightarrow \infty}\left\|p_{g, \mathcal{T}_{m}}^{(0)}-p_{g}^{(0)}\right\|_{\mathbf{L}^{\mathbf{2}}(\Omega)}=$ 0 and $\lim _{m \rightarrow \infty}\left\|p_{w, \mathcal{T}_{m}}^{(0)}-p_{w}^{(0)}\right\|_{\mathbf{L}^{2}(\Omega)}=0$.

If $p_{g, \mathcal{T}_{m}}$ and $p_{w, \mathcal{T}_{m}}$ fulfill the scheme (3.1) for all $m \in \mathbb{N}$, then there exist $p_{g} \in \mathbf{L}^{2}\left(Q_{T}\right)$, $p_{w} \in \mathbf{L}^{\mathbf{2}}\left(Q_{T}\right)$ and $q=p_{w}-p_{g}$ such that (up to a subsequence), letting $m \rightarrow \infty$,

1. $\left(p_{g}, \mathcal{T}_{m}\right)_{m \in \mathbb{N}}$ converges weakly in $\mathbf{L}^{2}\left(Q_{T}\right)$ to $p_{g} \in \mathbf{L}^{\mathbf{2}}\left((0, T) ; \mathbf{H}_{\mathbf{0}}^{\mathbf{1}}(\Omega)\right)$ and $\left(\nabla_{m} p_{g, \mathcal{T}_{m}}\right)_{m \in \mathbb{N}}$ converges weakly in $\mathbf{L}^{2}\left(Q_{T}\right)^{d}$ to $\nabla p_{g}$,

2. $\left(p_{w, \mathcal{T}_{m}}\right)_{m \in \mathbb{N}}$ converges weakly in $\mathbf{L}^{\mathbf{2}}\left(Q_{T}\right)$ to $p_{w} \in \mathbf{L}^{\mathbf{2}}\left((0, T) ; \mathbf{H}_{\mathbf{0}}^{\mathbf{1}}(\Omega)\right)$ and $\left(\nabla_{m} p_{w}, \mathcal{T}_{m}\right)_{m \in \mathbb{N}}$ converges weakly in $\mathbf{L}^{2}\left(Q_{T}\right)^{d}$ to $\nabla p_{w}$,

3. $\left(S\left(q_{\mathcal{T}_{m}}\right)\right)_{m \in \mathbb{N}}$ converges in $\mathbf{L}^{2}\left(Q_{T}\right)$ to $\widehat{S}:=S(q)$,

4. $\left(X\left(p_{g, \mathcal{T}_{m}}\right)\right)_{m \in \mathbb{N}}$ converges in $\mathbf{L}^{\mathbf{2}}\left(Q_{T}\right)$ to $\widehat{X}:=X\left(p_{g}\right)$,

5. $\left(K_{w, \mathcal{T}_{m}}\right)_{m \in \mathbb{N}}$ converges in $\mathbf{L}^{2}\left(Q_{T}\right)$ to $k_{w}(S(q))$ and $\left(K_{g, \mathcal{T}_{m}}\right)_{m \in \mathbb{N}}$ converges in $\mathbf{L}^{2}\left(Q_{T}\right)$ to $k_{g}(S(q))$,

6. $\left(\Psi_{\mathcal{T}_{m}}\right)_{m \in \mathbb{N}}$ converges in $\mathbf{L}^{2}\left(Q_{T}\right)$ to $X\left(p_{g}\right)$.

and $\left(p_{g}, p_{w}\right)$ is a weak solution of problem (1.2).

Proof. Thanks to Lemmas 3.2 and 3.5, we extract a subsequence such that items 1 and 2 of the theorem hold (we then denote $q_{\mathcal{T}_{m}}=p_{w, \mathcal{T}_{m}}-p_{g, \mathcal{T}_{m}}$ ). We now apply Lemma 3.6 , which ensures the existence of a subsequence, extracted from the preceding one, and of $\widehat{S}, \widehat{X}_{S} \in \mathbf{L}^{2}\left(Q_{T}\right)$, such that $\left(S\left(q_{\mathcal{T}_{m}}\right)\right)_{m \in \mathbb{N}}$ converges in $\mathbf{L}^{2}\left(Q_{T}\right)$ to $\widehat{S}$, and $\left(S\left(q_{\mathcal{T}_{m}}\right) X\left(p_{g, \mathcal{T}_{m}}\right)\right)_{m \in \mathbb{N}}$ converges in $\mathbf{L}^{2}\left(Q_{T}\right)$ to $\widehat{X}_{S}$. Using $S_{\text {min }} \leq S\left(q_{\mathcal{T}_{m}}\right)$. Thanks to Lemma 3.7 applied with $F=S$ and $u_{m}=q_{\mathcal{T}_{m}}$, we obtain that $\widehat{S}=S(q)$, and item 3 is proved. Using $S_{\min } \leq S\left(q_{\mathcal{T}_{m}}\right)$, we get that $X\left(p_{g, \mathcal{T}_{m}}\right)=\left(S\left(q_{\mathcal{T}_{m}}\right) X\left(p_{g, \mathcal{T}_{m}}\right)\right) / S\left(q_{\mathcal{T}_{m}}\right)$ converges in $\mathbf{L}^{2}\left(Q_{T}\right)$ to $\widehat{X}:=\widehat{X}_{S} / \widehat{S}$. Again applying Lemma 3.7 applied with $F=X$ and $u_{m}=p_{g, \mathcal{T}_{m}}$, we conclude that $\widehat{X}=X\left(p_{g}\right)$, thus concluding item 4 .

The application of Lemma 3.8 provides item 5 and that of Lemma 3.9 provides item 6 .

Let now be $\varphi \in \mathbf{C}_{\mathbf{c}}^{\infty}([0, T))$ and $\omega \in \mathbf{C}_{\mathbf{c}}^{\infty}(\Omega)$ two test functions. Furthermore, writing $\mathcal{T}$ instead of $\mathcal{T}_{m}$ and dropping the index $m$ wherever confusion is excluded, we define the approximations $\omega_{\mathcal{T}}$ and $\hat{\nabla}_{\mathcal{T}} \omega_{\mathcal{T}}$ of $\omega$ and $\nabla \omega$ by

$$
\begin{aligned}
& \omega_{\mathcal{T}}=\sum_{K \in \mathcal{T}} \omega\left(x_{K}\right) \chi_{K} \\
& \hat{\nabla}_{\mathcal{T}} \omega_{\mathcal{T}}=\sum_{\mathcal{D}_{K \mid L}}\left(\frac{\omega\left(x_{L}\right)-\omega\left(x_{K}\right)}{d_{K \mid L}} n_{K \mid L}+\nabla_{K \mid L, t} \omega\right) \chi_{\mathcal{D}_{K \mid L}}+\sum_{\mathcal{D}_{K \mid \sigma}}\left(\frac{\omega\left(x_{K}\right)}{d_{K \mid \sigma}} n_{K \mid \sigma}+\nabla_{K \mid \sigma, t} \omega\right) \chi_{\mathcal{D}_{K \mid \sigma}},
\end{aligned}
$$

where $\nabla_{K \mid L, t}$ denotes any tangential part of the discrete gradient in the diamond $\mathcal{D}_{K \mid L}$. It is worth to note that the operator $\widehat{\nabla}_{\mathcal{T}}$ has two important differences with $\nabla_{\mathcal{T}}$ defined by 
3.10 (there is no factor $d$ and there is a tangential component). Analogously, we define the approximation $\varphi_{\mathcal{T}}$ of $\varphi$ by

$$
\varphi_{\mathcal{T}}=\sum_{n=0}^{N-1} \varphi\left(t^{(n)}\right) \quad \delta_{\mathcal{T}} \varphi_{\mathcal{T}}=\sum_{n=0}^{N-1} \frac{\varphi\left(t^{n+1}\right)-\varphi\left(t^{(n)}\right)}{\delta t^{\left(n+\frac{1}{2}\right)}}
$$

Note that by consistency of the approximation $\omega_{\mathcal{T}}$ converges in $\mathbf{L}^{2}(\Omega)$ to $\omega$ and $\nabla_{m} \omega_{\mathcal{T}}$ converges in $\mathbf{L}^{2}(\Omega)$ to $\nabla \omega$. Analogously, $\varphi_{\mathcal{T}}$ converges in $\mathbf{L}^{2}([0, T))$ to $\varphi$ and $\delta_{\mathcal{T}} \varphi_{\mathcal{T}}$ converges in $\mathbf{L}^{2}([0, T))$ to $\varphi^{\prime}$.

Multiplying (3.1a) by $\delta t^{\left(n+\frac{1}{2}\right)} \varphi\left(t^{(n)}\right) \omega\left(x_{K}\right)$ and summing over all $K$ and $n$, we obtain

$$
\begin{aligned}
& \sum_{n=0}^{N-1} \sum_{K \in \mathcal{T}} \Phi_{K}\left(S_{K}^{(n+1)}-S_{K}^{(n)}\right) \varphi\left(t^{(n)}\right) \omega\left(x_{K}\right) \\
& \quad-\sum_{n=0}^{N-1} \delta t^{\left(n+\frac{1}{2}\right)} \varphi\left(t^{(n)}\right)\left[p_{w}^{(n+1)}, \omega_{\mathcal{T}}\right]_{k_{w}^{(n+1)}, \mathcal{T}}=\sum_{n=0}^{N-1} \delta t^{\left(n+\frac{1}{2}\right)} \varphi\left(t^{(n)}\right) f_{w, K}^{(n+1)} \omega\left(x_{K}\right) .
\end{aligned}
$$

Reordering of the terms in (3.16a) yields

$$
\begin{aligned}
\sum_{n=0}^{N-1} & \sum_{K \in \mathcal{T}} \Phi_{K}\left(S_{K}^{(n+1)}-S_{K}^{(n)}\right) \varphi\left(t^{(n)}\right) \omega\left(x_{K}\right) \\
& =-\sum_{n=0}^{N-1}\left(\varphi\left(t^{(n+1)}\right)-\varphi\left(t^{(n)}\right) \sum_{K \in \mathcal{T}} \Phi_{K} S_{K}^{(n+1)} \omega\left(x_{K}\right)-\varphi(0) \sum_{K \in \mathcal{T}} \Phi_{K} S_{K}^{(0)} \omega\left(x_{K}\right)\right. \\
& =-\int_{0}^{T} \delta_{\mathcal{T}} \varphi_{\mathcal{T}} \int_{\Omega} \Phi(x) S_{\mathcal{T}} \omega_{\mathcal{T}} d x d t-\varphi(0) \int_{\Omega} \Phi(x) S\left(q_{\mathcal{T}}^{(0)}\right) \omega_{\mathcal{T}} d x .
\end{aligned}
$$

And therefore

$$
\begin{aligned}
\lim _{m \rightarrow \infty} \sum_{n=0}^{N-1} \sum_{K \in \mathcal{T}} \Phi_{K} \delta_{\mathcal{T}}^{\left(n+\frac{1}{2}\right)} S_{K} \delta t^{\left(n+\frac{1}{2}\right)} \varphi\left(t^{(n)}\right) \omega\left(x_{K}\right) \\
\quad=-\int_{0}^{T} \varphi^{\prime}(t) \int_{\Omega} \Phi(x) S(q(x)) \omega(x) d x d t-\varphi(0) \int_{\Omega} \Phi(x) S\left(q^{(0)}(x)\right) \omega(x) d x .
\end{aligned}
$$

We then remark that, since the weak gradient defined by (3.10) contains only a component normal to the edges, the term in $(3.16 \mathrm{~b})$ can be rewritten as

$$
\sum_{n=0}^{N-1} \delta t^{\left(n+\frac{1}{2}\right)} \varphi\left(t^{(n)}\right)\left[p_{w}^{(n+1)}, \omega_{\mathcal{T}}\right]_{k_{w}^{(n+1)}, \mathcal{T}}=\int_{0}^{T} \varphi_{\mathcal{T}}(t) \int_{\Omega} K_{w, \mathcal{T}} \nabla_{m} p_{w} \hat{\nabla}_{\mathcal{T}} \omega_{\mathcal{T}} d x d t
$$

We easily have

$$
\lim _{m \rightarrow \infty} \sum_{n=0}^{N-1} \delta t^{\left(n+\frac{1}{2}\right)} \varphi\left(t^{(n)}\right) f_{w, K}^{(n+1)} \omega\left(x_{K}\right)=\int_{0}^{T} \varphi(t) \int_{\Omega} f_{w} \omega d x d t,
$$

and, due to the strong convergence of $K_{w, \mathcal{T}} \widehat{\nabla}_{\mathcal{T}} \omega_{\mathcal{T}}$ to $k_{w}(S(q)) \nabla \omega$ (using the $L^{\infty}$ bound of $K_{w, \mathcal{T}}$ ), and the weak convergence of $\nabla_{m} p_{w}$ to $\nabla p_{w}$, we have

$$
\lim _{m \rightarrow \infty} \int_{0}^{T} \varphi_{\mathcal{T}}(t) \int_{\Omega} K_{w, \mathcal{T}} \nabla_{m} p_{w} \widehat{\nabla}_{\mathcal{T}} \omega_{\mathcal{T}} d x d t=\int_{0}^{T} \varphi(t) \int_{\Omega} k_{w}(S(q)) \nabla p_{w} \nabla \omega d x d t
$$


which shows the convergence of (3.1a) to (1.2b). To show that (3.1b) converges to (1.2c), we follow the same steps as above.

Remark 3.11. We only state a weak convergence result for the pressures in Theorem 3.10. A stronger convergence result could probably be expected by passing to the limit $m \rightarrow \infty$ in (3.5) and comparing this limit with the equation obtained by taking the solution $p_{w}, p_{g}$ as test functions in (1.2). But such a work implies quite long developments due to the need of regularizing the test functions.

\section{Numerical examples}

To conclude the paper, we present two numerical example in one space dimension using the following parameter.

$$
\begin{array}{ll}
\Phi(x)=0.2 & \Lambda(x)=10^{-12} \\
\rho_{w}=10^{3} \frac{\mathrm{kg}}{\mathrm{m}^{3}} & \rho_{g}=10 \frac{\mathrm{kg}}{\mathrm{m}^{3}} \\
f_{w}(t, x)=0 & f_{g}(t, x)=0 \\
D=0 . &
\end{array}
$$

This means that we examine the results obtained in the case where the gas diffusion in the water phase is neglected. Furthermore, we specify the functions $S, X, k_{g}$ and $k_{w}$ as follows.

$$
\begin{array}{lll}
S(q)=\frac{1}{\left(\left(\frac{\max \{-q, 0\}}{p_{0}}\right)^{n}+1\right)^{m}} & \text { with } & n=2, m=1 / 3, p_{0}=10^{5} \mathrm{~Pa} \\
X\left(p_{g}\right)=\min \left\{c_{p} p_{g}, \bar{X}\right\} & \text { with } & \bar{X}=0.1 \\
k_{w}(S)=\max \left\{\frac{S^{2}}{\mu_{w}}, 10^{-5}\right\} & \text { with } & \mu_{w}=10^{-3} \\
k_{g}(S)=\max \left\{\frac{(1-S)^{2}}{\mu_{g}}, 10^{-5}\right\} & \text { with } & \mu_{g}=10^{-5} .
\end{array}
$$

Both test cases will be performed with the same initial and boundary data, given by

$$
\begin{array}{ll}
p_{g}(0, x)=10^{5} \mathrm{~Pa} & p_{w}(0, x)=10^{5} \mathrm{~Pa} \\
p_{g}(t, 0)=3 \cdot 10^{5} \mathrm{~Pa} & p_{g}(t, L)=10^{5} \mathrm{~Pa} \\
p_{w}(t, 0)=10^{5} \mathrm{~Pa} & p_{w}(t, L)=10^{5} \mathrm{~Pa} .
\end{array}
$$

To apply the algorithm (3.1), we define the values $S_{K \mid L}$ and $S_{K \mid \sigma}$ at the cell boundaries by

$$
S_{K \mid L}=\frac{1}{2}\left(S_{K}+S_{L}\right) \quad S_{K \mid \sigma}=\frac{1}{2}\left(S_{K}+S_{\sigma}\right),
$$

with $S_{K}=S\left(q_{K}\right)=S\left(p_{w, K}-p_{g, K}\right)$ and $S_{\sigma}=S\left(q_{\sigma}\right)$. Hereby, $q_{\sigma}$ denotes the value of $q$ at the boundary, specified by $q(t, 0)$ or $q(t, L)$ respectively. Note that due to the initial conditions we have $S^{(0)}:=S\left(q^{(0)}\right)=1$ in the whole computation domain. To take into account that this 
choice does not imply $k_{i}(S) \geq k_{i}^{\text {min }}>0$ for the standard choices of $k_{i}$, we bound $k_{i}$ artificially from below by $10^{-5}$. All convergence results continue to hold in this case, provided $S>0$ is satisfied during the computations.

For the first test case, we define the parameter $c_{p}$ of the function $X\left(p_{g}\right)$ by $c_{p}=3 \cdot 10^{-7}$ and assume a length of the spacial interval of $L=10^{3} \mathrm{~m}$. Figure 4 shows the distribution of $S, X\left(p_{g}\right), p_{g}$ and $p_{w}$ at different times $t$. Each line corresponds to a time step of $d t=1$ day, the simulation ends at time $T=15$ days. In this example, the parameter $c_{p}$ is chosen such
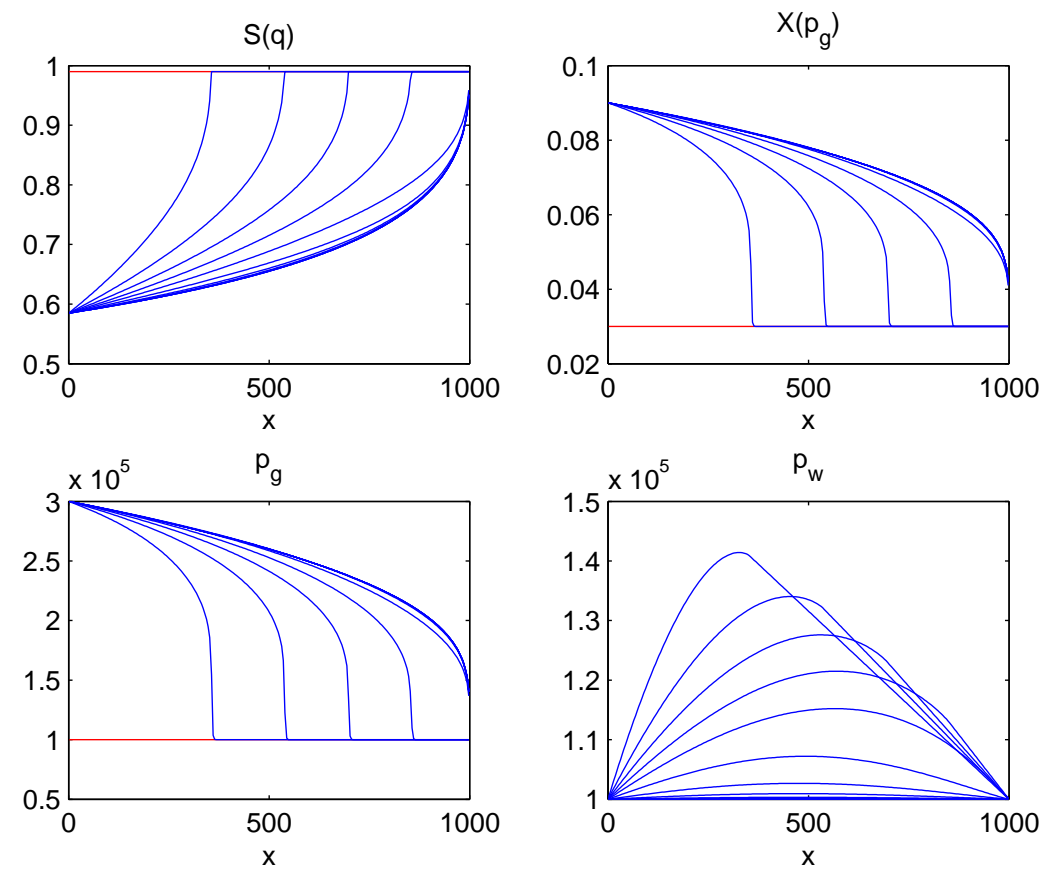

Figure 4: Test case 1: $X$ does not attain its maximum value.

that the maximum fraction of dissolved gas in the water phase is not attained, $X\left(p_{g}\right)$ is therefore less than $\bar{X}$ in the whole computational domain $[0, L] \times[0, T]$. Table 1 illustrates the convergence behavior of the variable $p_{g}$ for a sequence of different grid sizes. Therein, we use a fine grid solution $p_{g}^{r e f}(T)$ computed on a grid of $K=240$ cells as reference solution up to time $T=2$ years. The comparison is done by averaging of the reference solution on the coarse grid, i.e. we take the mean value of the fine grid solution on each coarse grid cell. The simulations are scaled to the unit intervals in space and time, such that the definition of the time step size $d t=d x^{2}$ in relation to the space discretization is meaningful. Furthermore, the pressure variables where scaled by $10^{5}$, i.e. $p_{g}=\tilde{p}_{g} \cdot 10^{5}$.

\begin{tabular}{|c||c|c|c|}
\hline $\mathrm{K}$ & 30 & 60 & 120 \\
\hline$\left\|\tilde{p}_{g}(1)-\tilde{p}_{g}^{\text {ref }}(1)\right\|_{L^{2}([0,1])}$ & $2.992 \cdot 10^{-3}$ & $1.430 \cdot 10^{-3}$ & $0.587 \cdot 10^{-3}$ \\
\hline
\end{tabular}

Table 1: Convergence study for the gas pressure in experiment 1 for the grid sizes $K=30,60$ and 120 .

Note that we do not report the convergence study for $p_{w}$ since it tends to a constant in 
the stationary solution and the deviations of the numerical solutions from this constant in the numerical experiments are all below the accuracy of the simulations.

For the second test case, we chose $c_{p}=3.5 \cdot 10^{-7}$, and a length of the domain of $L=10^{4}$. In this case, the maximum value of $X$ is attained. Figure 5 shows the distribution of $S$, $X\left(p_{g}\right), p_{w}$ and $p_{g}$ for time steps of $d t=10$ days, the simulation ends after $T=150$ days.
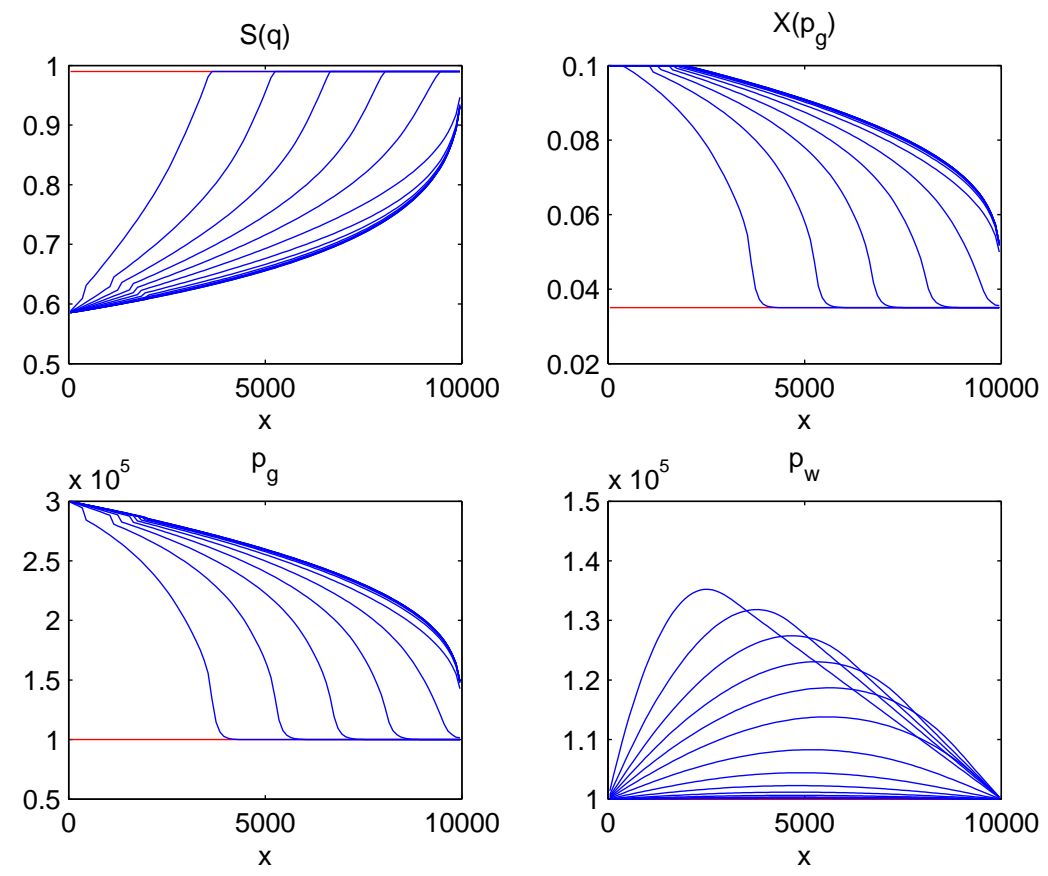

Figure 5: Test case 2: The maximum value of $X$ is attained.

In both test cases, we observe the profiles of the water pressure. They show a maximum value inside the domain, due to the fact that some water has to be removed (by flows at the two boundaries) in order to leave some porous volume for the apparition of the gas phase. As long as the gas phase does not appear, the gas pressure remains equal to its initial value, which simply corresponds to the initial gas concentration in the water phase (this remains exactly true since we performed the simulations with the value $D=0$ ). The gas pressure leaves its initial value mainly in the cells where the gas phase appears. We also remark, in the second case, that the moving front defined as the boundary of the domain $X\left(p_{g}\right)<\bar{X}$ has some influence on the profile of the saturation and of the gas pressure.

\section{Conclusion}

In this paper we have studied the convergence of a finite volume scheme with two-point flux approximation, to a solution of two-phase flow in porous media in the case of the partial dissolution of one phase into the other one. It is interesting to notice that the scheme which has been chosen here was the only one on which we have been able to derive this study, due to the need of nonlinear expressions of the primary unknowns as test functions. Further works to overcome this difficulty seem to be necessary, although it is not clear whether they can be 
fruitful.

\section{Acknowledgment}

The second author gratefully acknowledges the financial support by the German Research Foundation (DFG) in the framework of the Collaborative Research Center Transregio 75 Droplet Dynamics under Extreme Ambient Conditions and by the Landesstiftung BadenWürttemberg in the framework of the Eliteprogramm for Postdocs.

\section{References}

[1] M. Bendahmane, Z. Khalil, and M. Saad. Convergence of a finite volume scheme for gas water flow in a multi-dimensional porous media. arXiv preprint arXiv:1102.0582, 2011.

[2] K. Brenner, C. Cancès, and D. Hilhorst. A convergent finite volume scheme for two-phase flows in porous media with discontinuous capillary pressure field. In Finite volumes for complex applications. VI. Problems \& perspectives. Volume 1, 2, volume 4 of Springer Proc. Math., pages 185-193. Springer, Heidelberg, 2011.

[3] F. Caro, B. Saad, and M. Saad. Study of degenerate parabolic system modeling the hydrogen displacement in a nuclear waste repository. arXiv preprint arXiv:1202.3820, 2012.

[4] F. Caro, B. Saad, and M. Saad. Two-component two-compressible flow in a porous medium. Acta Appl. Math., 117:15-46, 2012.

[5] F. Z. Daïm, R. Eymard, and D. Hilhorst. Existence of a solution for two phase flow in porous media: the case that the porosity depends on the pressure. J. Math. Anal. Appl., 326(1):332-351, 2007.

[6] J. Droniou, T. Gallouët, and R. Herbin. A finite volume scheme for a noncoercive elliptic equation with measure data. SIAM J. Numer. Anal., 41(6):1997-2031 (electronic), 2003.

[7] G. Enchéry, R. Eymard, and A. Michel. Numerical approximation of a two-phase flow problem in a porous medium with discontinuous capillary forces. SIAM J. Numer. Anal., 43(6):2402-2422 (electronic), 2006.

[8] R. Eymard, T. Gallouët, and R. Herbin. Finite volume methods. In P. G. Ciarlet and J.-L. Lions, editors, Techniques of Scientific Computing, Part III, Handbook of Numerical Analysis, VII, pages 713-1020. North-Holland, Amsterdam, 2000.

[9] R. Eymard, C. Guichard, R. Herbin, and R. Masson. Gradient schemes for two-phase flow in heterogeneous porous media and the richards equation. ZAMM, to appear.

[10] R. Eymard, R. Herbin, and A. Michel. Mathematical study of a petroleum-engineering scheme. M2AN Math. Model. Numer. Anal., 37(6):937-972, 2003.

[11] G. Gagneux and M. Madaune-Tort. Analyse mathématique de modèles non linéaires de l'ingénierie pétrolière, volume 22 of Mathématiques \& Applications (Berlin) [Mathematics \& Applications]. Springer-Verlag, Berlin, 1996. With a preface by Charles-Michel Marle. 